\title{
Microscopic theory of black hole superradiance
}

\author{
Óscar J. C. Dias, ${ }^{1, *}$ Roberto Emparan, ${ }^{1,2, \dagger}$ and Alessandro Maccarrone ${ }^{1, \dagger}$ \\ ${ }^{1}$ Departament de Física Fonamental, Universitat de Barcelona, Marti i Franquès 1, E-08028 Barcelona, Spain \\ ${ }^{2}$ Institució Catalana de Recerca i Estudis Avançats (ICREA), Passeig Lluis Companys 23, 08010 Barcelona, Spain
}

(Received 26 December 2007; published 14 March 2008)

\begin{abstract}
We study how black hole superradiance appears in string microscopic models of rotating black holes. In order to disentangle superradiance from finite-temperature effects, we consider an extremal, rotating D1D5-P black hole that has an ergosphere and is not supersymmetric. We explain how the microscopic dual accounts for the superradiant ergosphere of this black hole. The bound $0<\omega<m \Omega_{H}$ on superradiant mode frequencies is argued to be a consequence of Fermi-Dirac statistics for the spin-carrying degrees of freedom in the dual conformal field theory. We also compute the superradiant emission rates from both sides of the correspondence, and show their agreement.
\end{abstract}

DOI: 10.1103/PhysRevD.77.064018

PACS numbers: 04.70.Dy, 11.25.Uv

\section{INTRODUCTION}

The microscopic string theory of black holes provides an accurate statistical counting of the Bekenstein-Hawking entropy [1-6] and a microscopic picture of Hawking radiation [7-11] at least for some classes of black holes. In the present paper we address how this microscopic theory also accounts for a characteristic phenomenon of rotating black holes: the black hole superradiance.

Superradiance is a phenomenon associated to the presence of an ergoregion around the black hole [12-19]. Since the Killing vector that defines the energy measured by asymptotic observers becomes spacelike within the ergosurface, it follows that in the ergoregion there can exist excitations with negative energy relative to infinity. So if we scatter a wave off the black hole, this wave can excite negative-energy modes that may subsequently fall into the horizon. To an asymptotic observer this will appear as a positive energy flux coming out of the horizon, and the scattered wave can emerge with higher amplitude than the impinging wave: this is known as superradiant scattering. If an incident wave $\Phi \sim f(r, \theta) e^{-i \omega t+i m \phi}$, with energy $\omega>0$ and angular momentum number $m$, scatters off a black hole with horizon angular velocity $\Omega_{H}$, the requirement that a negative-energy flux crosses the horizon towards the future is

$$
0<\omega<m \Omega_{H} .
$$

Only modes satisfying this condition can undergo superradiant amplification.

Superradiant scattering can be regarded as stimulated emission, and, just like the latter (classical) process is related by detailed balance to (quantum) spontaneous emission, rotating black holes are also known to spontaneously emit superradiant modes within the range (1.1), in a process closely related to Hawking radiation. These carry

\footnotetext{
*odias@ub.edu,

†emparan@ub.edu,

sandro@ffn.ub.es
}

away energy, but also angular momentum off the black hole. In our microscopic picture it is convenient to first describe the process of spontaneous superradiant emission, and then infer the stimulated emission.

When the black hole temperature is different from zero it is difficult to disentangle spontaneous superradiant emission from thermal Hawking radiation - in fact both become part of one and the same phenomenon. In this paper, however, we are interested only in the microphysics behind the presence of an ergoregion and the existence of superradiant modes (1.1). So we will investigate the spontaneous emission from an extremal, i.e., zero-temperature, rotating black hole, for which thermal Hawking radiation is absent. Since the black hole has a "cold" ergoregion, we refer to it as an ergo-cold black hole. This will allow us to isolate superradiance: only modes that satisfy (1.1) will be emitted. Note, however, that after the emission of superradiant quanta begins, the angular momentum will be reduced below its maximal value and the black hole will be driven away from extremality, so thermal Hawking radiation will promptly set in. It is the onset of the decay that will give us more neatly the microscopic basis of the superradiant bound (1.1).

There have been previous papers dealing with emission rates from rotating black holes and the microscopic calculations that match them $[9,18,20,21]$ (see [22,23] for a review), in some cases discussing, more or less directly, aspects of superradiance. Typically, these papers have computed the absorption cross sections for a nonextremal black hole and for its microscopic finite-temperature dual. Even if these results exhibit essential agreement between both sides, we feel that the long calculations involved, and the mixing with thermal Hawking radiation, hide some very simple microphysics behind (1.1). We hope to clarify the microscopic origin of the ergoregion and provide a simple interpretation of the superradiant modes in it. We shall follow mostly a suggestion advanced in [5], making it more precise and quantitative. A salient conclusion of our analysis is a clear understanding of the bound (1.1) as essentially a consequence of Fermi-Dirac statistics for 
the microscopic degrees of freedom that give the black hole its angular momentum.

The paper is structured as follows. The main ideas are introduced first in a fairly self-contained and elementary discussion, while the technically most involved analysis is postponed to later sections. So, Sec. II begins with a qualitative review of the microscopic model of D1-D5-P black holes, with and without ergospheres, and then proceeds to derive (1.1) from simple microscopic considerations. The detailed calculations of absorption rates, which are needed for other quantitative aspects of superradiance, are studied at the supergravity level in Sec. III. This is an extension of previous analyses of radiation from the D1D5-P black holes studied at length in [20,21]. We do generalize their results to include momentum for the bulk scalar. The microscopic side is then developed in Sec. IV. Here we first establish the details of the identification of the dual conformal field theory (CFT) state, compute the microscopic absorption cross section, and compare to the supergravity results. Section V concludes with a qualitative discussion of how our picture accounts for superradiance in other systems with "cold ergoregions." The appendix contains an analysis of how the near-horizon geometry encodes information about the possibility of superradiance in the full geometry.

\section{MICROPHYSICS OF COLD ERGOREGIONS}

We begin by introducing the microscopic picture of superradiance and then provide a simple derivation and interpretation of the bound (1.1) for the ergo-cold black hole.

\section{A. Qualitative microscopic origin of the ergoregion}

Our basic picture applies to any black hole that admits an "effective string" description, i.e., to which $\mathrm{AdS}_{3} / \mathrm{CFT}_{2}$ duality applies, ${ }^{1}$ but for definiteness we focus, for the most part, on the D1-D5-P system, which describes a class of near-supersymmetric five-dimensional black holes. We shall begin by reviewing in qualitative terms the microscopic picture of several kinds of D1-D5-P black holes.

The D1 and D5-branes form a bound state whose lowenergy dynamics is described by a $1+1$-dimensional field theory along their common worldvolume directions (the other four directions wrap a small $T^{4}$ or $K 3$ ). It is a nonchiral conformal field theory with $(4,4)$ supersymmetry, i.e., both the left- and right-moving sectors are supersymmetric. Supersymmetry itself will not play any essential role in our discussion, but the existence of fermionic excitations in at least one of the two chiral sectors is important. For large numbers $N_{1}, N_{5}$, of D1 and D5 branes, the

\footnotetext{
${ }^{1}$ And even to some that may not, like in [5], although in this case the bound (1.1) is recovered only up to numerical factors.
}

central charge of both sectors is $c=6 N_{1} N_{5}$. The CFT can have left- and right-moving excitations, with levels $L_{0}$ and $\bar{L}_{0}$, corresponding to open string excitations propagating along the worldvolume of the branes. These give rise to a linear momentum $P$.

When the spatial direction along this D1-D5-P system is compactified on a circle of size $2 \pi R$ (much larger than the other compact directions), we obtain a five-dimensional configuration. Typically, the state corresponding to a black hole has both sectors populated by thermal ensembles of excitations with temperatures $T_{L}$ and $T_{R}$. If the two sectors interact only very weakly, the total entropy, energy, and momentum are $S=S_{L}+S_{R}, E=P_{L}+P_{R}$, and $P=$ $P_{L}-P_{R}$, with quantized momenta $P_{L, R}=N_{L, R} / R$. Since $T_{L, R}^{-1}=\left(\partial S_{L, R} / \partial P_{L, R}\right)=2\left(\partial S_{L, R} / \partial E\right)_{P}$, it follows that the actual temperature $T_{H}^{-1}=(\partial S / \partial E)_{P}$ of the entire configuration is

$$
T_{H}^{-1}=\frac{1}{2}\left(T_{L}^{-1}+T_{R}^{-1}\right) .
$$

If any of the two sectors is in a ground state (either $T_{L}$ or $T_{R}$ vanish), the temperature of the entire system vanishes.

The simplest black hole corresponds to a thermal ensemble of excitations in only one of the two sectors, say the right-moving one. Supersymmetry of the left sector is then preserved, and $T_{L}=T_{H}=0$. This is the static supersymmetric extremal black hole of Ref. [1] (see Fig. 1(a)). If both sectors are excited, then generically the system has $T_{H} \neq 0$. An open string excitation from the left sector can combine with an open string from the right sector, and form a closed string that propagates away into the bulk of spacetime (see Fig. 1(b)). This is the microscopic counterpart of the Hawking emission at temperature $T_{H}[2,24]$.

To include rotation, we take into account that the fermionic excitations of the left and right sectors are charged under the R-symmetry group $S U(2)_{L} \times S U(2)_{R}$ of the supersymmetric CFT. These R-symmetries generate the five-dimensional spatial rotation group $S O(4) \simeq S U(2)_{L} \times$ $S U(2)_{R}$. So the R-charge corresponds to spacetime angular momentum, $J_{L}$ or $J_{R}$, respectively, for left and right fermions. If many of these fermions are coherently polarized we obtain a macroscopically large angular momentum. This projection into definite polarization shifts the levels as

$$
L_{0}=N_{L}-\frac{6 J_{L}^{2}}{c}, \quad \bar{L}_{0}=N_{R}-\frac{6 J_{R}^{2}}{c},
$$

and, in particular, the total entropy and temperature are reduced.

Observe now that there are two distinct ways of achieving an extremal $\left(T_{H}=0\right)$ rotating black hole. In the first one we set, say, $N_{L}=0=J_{L}$ (so half of the supersymmetry is preserved), $\bar{L}_{0}>0$, and some of the right-moving fermions polarized to give $J_{R} \neq 0$ [25]. Since only one of the two sectors is excited, the left- and right-moving open strings cannot combine to emit a closed string (see Fig. 1(c)). This fits nicely with the property that the horizon 
(a)

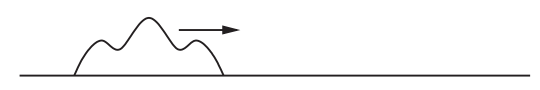

(c)

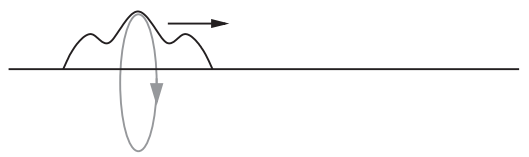

(b)

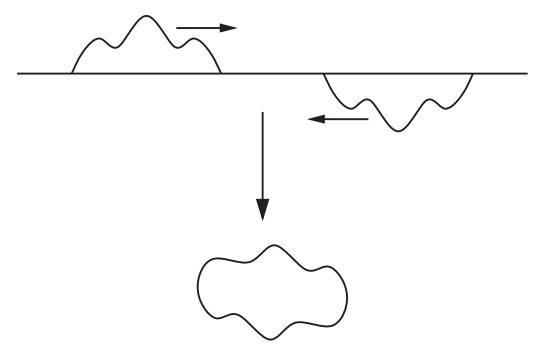

(d)

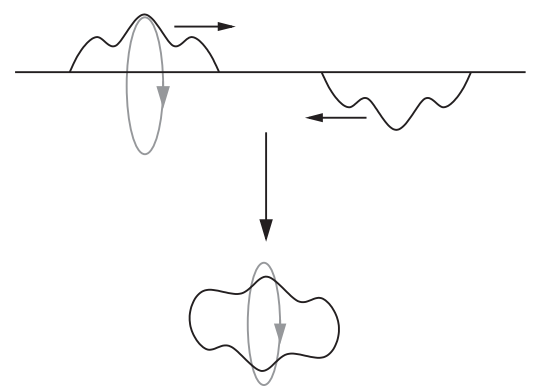

FIG. 1. Four different kinds of black hole in the "effective string" picture. The excitations of the two chiral sectors, with levels $L_{0}$ (left-moving) and $\bar{L}_{0}$ (right-moving), correspond to open strings attached to the brane bound state. (a) Supersymmetric static black hole: $L_{0}=0, \bar{L}_{0}=N_{R}$ : only the right-moving sector is excited. (b) Near-supersymmetric static black hole: $L_{0}=N_{L}>0, \bar{L}_{0}=$ $N_{R}>0$. Left- and right-moving excitations can annihilate to emit a closed string: this is Hawking radiation. (c) Supersymmetric rotating black hole: $L_{0}=0, \bar{L}_{0}=N_{R}-6 J_{R}^{2} / c>0$. The coherent polarization of right-moving fermions yields a macroscopic (selfdual) angular momentum $J_{R}$. In the absence of left-moving open strings, there cannot be any radiation of closed strings, hence there is no Hawking nor superradiant emission. (d) Ergo-cold black hole: $L_{0}>0$, and $\bar{L}_{0}=N_{R}-6 J_{R}^{2} / c=0$ with $N_{R}>0$. The right-moving sector is a Fermi sea of polarized fermionic excitations, so the temperature vanishes. Open strings in this sector can interact with those in the left sector and emit closed strings that carry angular momentum: the black hole possesses a superradiant ergosphere. The superradiant bound on modes (1.1) is directly related to the energy of the Fermi level, and thus is a consequence of Fermi-Dirac statistics for the excitations of the CFT.

of the corresponding black hole remains static relative to asymptotic observers: since $\Omega_{L}=\Omega_{R}=0$ there is no ergosphere nor superradiant emission, even if $J_{R} \neq 0$.

The second, less studied way to achieve a zerotemperature rotating black hole is by having the rightmoving sector contain only polarized fermions that fill energy levels up until the Fermi level. This occurs when

$$
N_{R}=\frac{6 J_{R}^{2}}{c}
$$

This is a ground state, $\bar{L}_{0}=0$, at fixed $J_{R}$, with zero entropy and at zero temperature. The left-moving sector is assumed to be thermally excited, with $L_{0}>0$ : this provides for the entropy. Both sectors can carry angular momentum, so, in contrast to the supersymmetric case, the total angular momentum need not be self-dual nor antiself-dual. More importantly, even if the system is at zero temperature, both left- and right-moving open strings are present and can annihilate to emit a closed string (see Fig. 1(d)). Since the right-moving open string necessarily carries spin, so will also the emitted radiation. This is, qualitatively, what we expect from superradiant emission. In fact, the corresponding black hole possesses an ergosphere and superradiant emission is present. So we have found a qualitative microscopic picture for the superradiance from the ergo-cold black hole [5].

\section{B. Microscopic derivation of the superradiant frequency bound}

We can be more quantitative and recover the superradiant frequency bound from this microscopic picture. In five spacetime dimensions the black hole can rotate in two independent planes and if we label the rotation angles on these planes by $\phi$ and $\psi$ then the bound (1.1) is generalized to

$$
0<\omega<m_{\phi} \Omega_{\phi}+m_{\psi} \Omega_{\psi},
$$

where $\Omega_{\phi, \psi}$ are the horizon angular velocities on each rotation plane, and $m_{\phi, \psi}$ the corresponding angular mo- 
mentum ("magnetic") quantum numbers. We may instead use the left and right Euler angles $\psi_{L, R}=\phi \mp \psi$, in terms of which the bound is

$$
0<\omega<m_{L} \Omega_{L}+m_{R} \Omega_{R},
$$

with $m_{L, R}=\frac{1}{2}\left(m_{\phi} \mp m_{\psi}\right)$ and $\Omega_{L, R}=\Omega_{\phi} \mp \Omega_{\psi}$. This is slightly more convenient, since as we saw above these angles diagonalize the R-charges (i.e., target-space spins) of the left- and right-moving fermions of the CFT.

The ergo-cold black hole described above has $\Omega_{R} \neq 0$ and $\Omega_{L}=0$ (although $J_{L}$ need not vanish). So the bound is

$$
0<\omega<m_{R} \Omega_{R},
$$

i.e., $m_{L}$ does not limit the frequencies. We wish to derive Eq. (2.6) from our microscopic picture.

To begin with, we can easily obtain that at zero temperature only one of $\Omega_{L}, \Omega_{R}$, can be different from zero. The two sectors of the CFT have negligible interaction, so $S\left(E, P, J_{L}, J_{R}\right)=S_{L}\left(E_{L}, J_{L}\right)+S_{R}\left(E_{R}, J_{R}\right)$. For each sector we have a chemical potential $\mu_{L, R}$ associated to the respective R-charges, i.e., $J_{L, R}$, through

$$
\frac{\mu_{L, R}}{T_{L, R}}=-\left(\frac{\partial S_{L, R}}{\partial J_{L, R}}\right)_{E_{L, R}} .
$$

The angular velocities of the total system are in turn

$$
\frac{\Omega_{L, R}}{T_{H}}=-\left(\frac{\partial S\left(E, P, J_{L}, J_{R}\right)}{\partial J_{L, R}}\right)_{E, P},
$$

where $T_{H}$ is the total system's temperature (2.1). Hence

$$
\Omega_{L, R}=\frac{T_{H}}{T_{L, R}} \mu_{L, R},
$$

and in the extremal limit in which $T_{R} \rightarrow 0$,

$$
\Omega_{R} \rightarrow 2 \mu_{R}, \quad \Omega_{L} \rightarrow 0 .
$$

As we explained above, for the ergo-cold black hole we take the right sector of the CFT to be populated by polarized fermions filling up to the Fermi level, so their number density distribution is a step function

$$
\rho\left(\epsilon, j_{R}\right)=\Theta\left(j_{R} \mu_{R}-\epsilon\right) .
$$

Here $\epsilon$ is the energy and $j_{R}$ the R-charge of the fermion, i.e., spin in $S U(2)_{R}$, which in general can be $\pm 1 / 2$. We assume that in the state (2.11) they are all polarized with $j_{R}=+1 / 2$, to achieve maximum angular momentum; see (2.3). Using the chemical potential $\mu_{R}$ introduced above, the Fermi energy is

$$
\epsilon_{\mathrm{Fermi}}=\frac{\mu_{R}}{2}=\frac{\Omega_{R}}{4} .
$$

In this state it is possible to have a collision of left- and right-moving open strings creating a closed string massless scalar mode. Our aim is to show that if this scalar has frequency $\omega$ and angular momentum numbers $\ell, m_{R}$, and
$m_{L}$, then $\omega$ must lie in the range (2.6). In order for the scalar to escape to infinity its energy must be positive, so we need only derive the upper bound in (2.6).

The interaction vertex involves bosonic and fermionic open strings from each sector, in either the initial or final states. But the spin of the scalar is provided only by fermions. For a given $\ell$ the angular momentum of the scalar is in the $(\ell / 2, \ell / 2)$ representation of $S U(2)_{L} \times$ $S U(2)_{R}$, i.e., $\left|m_{L}\right|,\left|m_{R}\right| \leq \ell / 2$, so we need $\ell$ fermionic open strings from each sector to match the spin quantum numbers of the scalar. A minimal scalar at s-wave $(\ell=0)$ couples to an operator of conformal dimension $(1,1)$, typically of the form $\partial_{+} X \partial_{-} X$, i.e., one boson from each sector. Then, at the $\ell^{\text {th }}$ partial wave it will couple to this boson pair and to the $\ell$ fermion pairs. Additional bosons may be involved, but then the amplitudes are suppressed by higher powers of the coupling and the frequency, although we need not assume their absence.

For our system, the right-sector open strings in the initial state in the interaction can only be fermionic with $j_{R}=$ $+1 / 2$. The fermions in the final state can have either $j_{R}=$ $\pm 1 / 2$ : we take the numbers of each kind of these to be $n_{ \pm}$, so the number of initial fermions from the right sector is $\ell-n_{+}-n_{-}$. The balance of angular momentum in the interaction is then

$$
\frac{1}{2}\left(\ell-n_{+}-n_{-}\right)=m_{R}+\frac{1}{2} n_{+}-\frac{1}{2} n_{-},
$$

i.e., the closed string is emitted with

$$
m_{R}=\frac{\ell}{2}-n_{+} .
$$

We will not need to consider any specific properties of the left-moving modes in our analysis.

Both the left and right sectors contribute an equal amount $\omega / 2$ to the energy of the emitted closed string otherwise the latter would carry the difference as a net momentum: this more general case will be dealt with later below. The energy budget of the interaction in the right sector is then

$$
\omega_{R}^{(f) \text { in }}=\frac{\omega}{2}+\omega_{R}^{(f) \text { out }}+\omega_{R}^{(b)},
$$

where $f$ and $b$ denote fermionic and bosonic open strings. In the left-hand side of this equation we have the energy of the $\ell-n_{+}-n_{-}$initial fermions. Since their energy levels are bounded above by the Fermi energy (2.12), we have

$$
\omega_{R}^{(f) \text { in }} \leq\left(\ell-n_{+}-n_{-}\right) \epsilon_{\mathrm{Fermi}}=\left(\ell-n_{+}-n_{-}\right) \frac{\Omega_{R}}{4} .
$$

As for the final fermions, the energies of the $n_{-}$fermions with $j_{R}=-1 / 2$ are not constrained other than to be positive: they may fill states with less or more energy 
than $\epsilon_{\mathrm{Fermi}}$. But the $n_{+}$fermions with $j_{R}=+1 / 2$ must have energies above the Fermi level, since in the initial configuration the levels below $\epsilon_{\mathrm{Fermi}}$ are all filled with positive-spin fermions. This sets a lower bound

$$
\omega_{R}^{(f) \text { out }}>n_{+} \frac{\Omega_{R}}{4} .
$$

The energy of the bosonic open strings is only constrained to be positive, $\omega_{R}^{(b)}>0$. Then, Eq. (2.15), together with (2.14), (2.16), and (2.17), yields the inequality

$$
\omega<m_{R} \Omega_{R}-\frac{n_{-}}{2} \Omega_{R} \leq m_{R} \Omega_{R},
$$

which reproduces exactly the superradiant bound (2.6) derived for the rotating black hole. ${ }^{2}$ Note that this result follows essentially from Pauli's exclusion principle for the polarized fermions in the initial state: the superradiant bound on frequencies is a consequence of Fermi-Dirac statistics for the carriers of angular momentum in the dual CFT.

Note that at least one bosonic open string must appear in the right sector in the final state, so the system will not remain extremal after it begins to radiate. This is also just like we anticipated from the supergravity side.

The left-moving fermions, which can contribute arbitrarily to $m_{L}$, have not played any role in this derivation. This is in accord with the fact that when $\Omega_{L}=0$ (even if $\left.J_{L} \neq 0\right), m_{L}$ does not appear in the macroscopically derived bound (2.4).

\section{Four-dimensional black holes}

This analysis applies almost immediately to the fourdimensional black holes described by a dual chiral $(0,4)$ CFT. Only the right sector is supersymmetric so the Rsymmetry consists of a single $S U(2)$ group. This corresponds to the four-dimensional rotation group $S U(2) \simeq$ $S O(3)$. Again, non-Bogomol'nyi-Prasad-Sommerfield (BPS) extremal rotating black holes exist, with four charges, that possess an ergosphere and the accompanying superradiant modes satisfying (1.1). The dual microscopic state is essentially the same as above: the right sector is filled up to $\epsilon_{\mathrm{Fermi}}=\Omega_{H} / 4$ with fermions with $j=+1 / 2$, while the left sector is in a thermal ensemble and accounts for the entropy. The emission of a closed string massless scalar with quantum numbers $(\omega, \ell, m)$ involves $2 \ell$ rightsector fermions since now $|m| \leq \ell$. So (2.14) is replaced by

\footnotetext{
${ }^{2}$ The bound is as close as possible to saturation when $n_{-}=0$, the boson energy $\omega_{R}^{(b)}$ is minimal (set by the gap $\sim 1 / N_{1} N_{5} R$ ), and all fermions are the closest possible to the Fermi energy (i.e., within $\sim 1 / N_{1} N_{5} R$ of it). If $n_{-}>0$ then this closest value to the bound cannot be achieved.
}

$$
m=\ell-n_{+} .
$$

There is also one boson from the right sector in the final state of the interaction. From the left sector the only requirement is an operator of conformal dimension $\Delta_{L}=$ $1+\ell$. Following the steps above we find

$$
\omega<m \Omega_{H}-\frac{n_{-}}{2} \Omega_{H} \leq m \Omega_{H} .
$$

Thus Eq. (1.1) has been derived microscopically for this ergo-cold black hole.

\section{No superradiant emission of linear momentum}

We can also consider the emission of closed strings that carry away some of the momentum $P$ of the D1-D5-P system. This is also of interest, as the momentum corresponds to one of the three charges of the black hole and there is a charge-ergoregion associated to it. From the sixdimensional perspective, the horizon of the black string is moving with velocity $V_{H}$ along the string direction $y$, and the superradiance bound for a mode $\sim \exp (-i \omega t+i p y+$ $\left.i m_{L} \psi_{L}+i m_{R} \psi_{R}\right)$ is modified to

$$
p<\omega<m_{L} \Omega_{L}+m_{R} \Omega_{R}+p V_{H} .
$$

In the non-BPS extremal rotating limit that we study, the black hole has $\Omega_{L} \rightarrow 0$. For a generic D1-D5-P black hole the velocity is $\left|V_{H}\right| \leq 1$, but we are particularly interested in the decoupling limit in which the D1 and D5 charges of the black hole are much larger than its momentum or the energy above the BPS bound. In this limit, the ergo-cold black hole has $V_{H} \rightarrow 1$, so the bound becomes

$$
0<\omega-p<m_{R} \Omega_{R} .
$$

We can easily derive this again from microscopic considerations. First note that the first law of thermodynamics gives

$$
\frac{V_{H}}{T_{H}}=-\left(\frac{\partial S}{\partial P}\right)_{E}
$$

Reasoning as we did when deriving (2.1) for a two-sector system, we find

$$
V_{H}=\frac{T_{H}}{2}\left(T_{R}^{-1}-T_{L}^{-1}\right)=\frac{T_{L}-T_{R}}{T_{L}+T_{R}},
$$

so $V_{H} \rightarrow 1$ when $T_{R} \rightarrow 0$. Also observe that in any case $\left|V_{H}\right| \leq 1$.

The left- and right-moving open strings that interact to emit a closed string of frequency $\omega$ and momentum $p$ do not in this case have the same energy, but instead

$$
\epsilon_{L, R}=\frac{\omega \pm p}{2} .
$$

We can follow now the same arguments for the right-sector dynamics that we used above, only changing $\omega / 2 \rightarrow \epsilon_{R}$. 
Hence we obtain

$$
\omega-p<m_{R} \Omega_{R} .
$$

In order to complete the derivation of (2.22) we need only notice that if the closed string is to arrive at infinity as an on-shell, propagating state, it must satisfy $\omega>0$ and $\omega^{2}-$ $p^{2} \geq 0$, i.e., $\omega \geq|p| \geq p$.

This implies that there cannot be any superradiant emission of linear momentum (i.e., $P$ charge in five dimensions) unless angular momentum is radiated as well. This is in spite of the fact that in the black hole geometry there is a momentum ergoregion, even in the absence of rotation. From the supergravity point of view, the reason for this difference between the emission of linear and angular momentum is that in the former case the contribution to the effective potential for scalar propagation coming from the momentum does not fall off at infinity but creates an asymptotic potential barrier of height $p$, so if $\omega<|p|$ the wave is asymptotically exponentially suppressed.

Put another way, in a Kaluza-Klein (KK) reduction to five dimensions the scalar has mass $|p|$ and a propagating wave at infinity must satisfy $\omega>|p|$. So a would-be superradiant momentum mode, satisfying $\omega<p V_{H}$, cannot escape to infinity since $V_{H} \leq 1$ : if emitted, it gets reflected back off to the black hole by the effective potential. In contrast, the centrifugal potential barriers fall off faster at large distances: the spin does not affect the dispersion relation of the wave at infinity. From the microscopic perspective, there is a possible interaction vertex for the emission of a scalar with linear momentum and zero angular momentum: take an initial state with only a leftmoving boson, and a final state with a right-moving boson and a bulk scalar. However, in this case the scalar would have $\omega<|p|$ and therefore could only exist as a virtual excitation. ${ }^{3}$

\section{E. Superradiant amplification, extremal and nonextremal}

We have obtained a microscopic picture for the spontaneous emission of superradiant scalars off an extremal nonBPS rotating black hole - the ergo-cold black hole. It is clear now that, if there is an incident flux of this scalar field on the black hole, then those modes that satisfy the bound (1.1) will undergo stimulated emission. This is simply the familiar phenomenon that the amplitude to emit a boson is amplified by a factor $\sqrt{N+1}$ if the state to which the system decays already contains $N$ bosons. That is, superradiant amplification follows conventionally from the relation between Einstein's $A$ and $B$ coefficients. For a

\footnotetext{
${ }^{3}$ An alternative interpretation is in terms of charge superradiance: an extremal Reissner-Nordstrom black hole can spontaneously emit particles of charge $e$ and mass $m$ only if $|e|>m$ [26]. In our case, the five-dimensional mass and KK electric charge of the particles are both equal to $p$.
}

classical incident wave, i.e., with large bosonic occupation number $N$, the stimulated emission is then a classical process.

In more detail, in our system at zero temperature we have argued that superradiant modes, and only them, can be emitted and have a finite decay rate $\Gamma_{\ell m}(\omega)$. Moreover, the system cannot absorb any superradiant mode: if in the argument that lead to the superradiant bound (2.6) we change the scalar from the final to the initial state, i.e., $\omega \rightarrow-\omega, m_{R} \rightarrow-m_{R}$, we see that absorption of this scalar can only happen when $\omega>m_{R} \Omega_{R}$. So, for an incident flux $\mathcal{F}_{\text {in }}$, detailed balance yields a total absorption cross section of superradiant modes $\sigma_{\ell m}(\omega)=$ $-\Gamma_{\ell m}(\omega) / \mathcal{F}_{\text {in }}<0$.

The absorption cross -section determines the ratio between the outgoing and ingoing fluxes as

$$
\frac{\mathcal{F}_{\text {out }}}{\mathcal{F}_{\text {in }}}=1-\frac{\omega^{3}}{(\ell+1)^{2} 4 \pi} \sigma_{\ell m}
$$

(this is the relation in five dimensions, see [11] for generic dimension). Superradiant modes, and only them, have $\sigma_{\ell m}<0$, and therefore yield $\mathcal{F}_{\text {out }}>\mathcal{F}_{\text {in }}$, as desired.

This argument shows that the extremal rotating system that we study exhibits classical stimulated amplification for those modes that it can spontaneously decay into, i.e., modes that satisfy (2.6). What happens away from extremality? In this case, the system can spontaneously emit modes of any frequency by the microscopic dual of Hawking radiation. Why, then, is there superradiant amplification only for modes that satisfy (2.6)? The reason is known: the first law, applied to an emission process from the black hole with $\delta E=-\omega$ and $\delta J=-m$, states that

$$
\frac{\kappa}{8 \pi G} \delta \mathcal{A}_{H}=-\left(\omega-m \Omega_{H}\right)
$$

Then, the classical stimulated emission of a mode with $\omega>m \Omega_{H}$ would violate the area law $\delta \mathcal{A}_{H} \geq 0$ [15]. So, classically, the emission of such nonsuperradiant modes is strictly forbidden, while microscopically it is allowed but statistically suppressed by a factor

$$
e^{\delta S}=e^{-\left(\omega-m \Omega_{H}\right) / T_{H}} .
$$

This is of course the Boltzmann factor for Hawking radiation.

Sometimes the existence of the superradiant frequency bound (1.1) is presented as a consequence of the area law. But we see that the latter is important only in constraining the classical, macroscopic process. Entropic considerations did not play any role in our microscopic analysis, which nevertheless shows that the superradiant bound on modes holds strictly at the microscopic level for emission at zero temperature. 


\section{EMISSION RATES: SUPERGRAVITY ANALYSIS}

The preceding analysis has provided a qualitative origin of the superradiant ergoregion in rotating black holes at zero temperature. We have also given a quantitative elementary derivation of the superradiant frequency bound. A more precise match between the two descriptions is obtained when one considers the actual emission rates.

To do so, in this section we carry out the supergravity computation of absorption cross sections and Hawking and superradiant emission rates for a minimal scalar. We consider the most general case where the black hole has all charges and rotations turned on, and the scalar has generic quantum numbers for the frequency, spins, and linear momentum along the $S^{1}$ string direction. At the end of the section we particularize to the ergo-cold black hole in order to isolate the effects of the ergosphere.

\section{A. The D1-D5-P family of black holes}

The D1-D5-P black hole solutions belong to type IIB supergravity compactified to five dimensions on $T^{4} \times S^{1}$. The $T^{4}$ is assumed to be much smaller than the $S^{1}$ so we view the system as a six-dimensional black string. The most general solution is described by eight parameters: a parameter $M_{0}$ that measures deviation away from supersymmetry; two spin parameters for rotation in two orthogonal planes, $a_{1}, a_{2}$; three "boost" parameters, $\delta_{1}, \delta_{5}, \delta_{p}$, which fix the D1-brane, D5-brane, and momentum charges, respectively; and two moduli: the radius $R$ of the $S^{1}$, and the volume $V$ of the $T^{4}$. We choose units such that the five-dimensional Newton constant is $G_{5}=$ $G_{10} / 2 \pi R V \equiv \pi / 4$.

The metric of the six-dimensional black string is $[21,27,28]$

$$
\begin{aligned}
d s^{2}= & -\frac{f}{\sqrt{H_{1} H_{5}}}\left(d t^{2}-d y^{2}\right)+\frac{M_{0}}{\sqrt{H_{1} H_{5}}}\left(s_{p} d y-c_{p} d t\right)^{2}+\sqrt{H_{1} H_{5}}\left(\frac{r^{2} d r^{2}}{\left(r^{2}+a_{1}^{2}\right)\left(r^{2}+a_{2}^{2}\right)-M_{0} r^{2}}+d \theta^{2}\right) \\
& +\left(\sqrt{H_{1} H_{5}}-\left(a_{2}^{2}-a_{1}^{2}\right) \frac{\left(H_{1}+H_{5}-f\right) \cos ^{2} \theta}{\sqrt{H_{1} H_{5}}}\right) \cos ^{2} \theta d \psi^{2}+\left(\sqrt{H_{1} H_{5}}+\left(a_{2}^{2}-a_{1}^{2}\right) \frac{\left(H_{1}+H_{5}-f\right) \sin ^{2} \theta}{\sqrt{H_{1} H_{5}}}\right) \sin ^{2} \theta d \phi^{2} \\
& -\frac{M_{0}}{\sqrt{H_{1} H_{5}}}\left(a_{1} \cos ^{2} \theta d \psi+a_{2} \sin ^{2} \theta d \phi\right)^{2}-\frac{2 M_{0} \cos ^{2} \theta}{\sqrt{H_{1} H_{5}}}\left[\left(a_{1} c_{1} c_{5} c_{p}-a_{2} s_{1} s_{5} s_{p}\right) d t+\left(a_{2} s_{1} s_{5} c_{p}-a_{1} c_{1} c_{5} s_{p}\right) d y\right] d \psi \\
& -\frac{2 M_{0} \sin ^{2} \theta}{\sqrt{H_{1} H_{5}}}\left[\left(a_{2} c_{1} c_{5} c_{p}-a_{1} s_{1} s_{5} s_{p}\right) d t+\left(a_{1} s_{1} s_{5} c_{p}-a_{2} c_{1} c_{5} s_{p}\right) d y\right] d \phi
\end{aligned}
$$

where we use the notation $c_{i} \equiv \cosh \delta_{i}, s_{i} \equiv \sinh \delta_{i}$, and

$$
\begin{aligned}
f(r) & =r^{2}+a_{1}^{2} \sin ^{2} \theta+a_{2}^{2} \cos ^{2} \theta, \\
H_{i}(r) & =f(r)+M_{0} s_{i}^{2}, \quad \text { with } i=1,5, \\
g(r) & =\left(r^{2}+a_{1}^{2}\right)\left(r^{2}+a_{2}^{2}\right)-M_{0} r^{2} .
\end{aligned}
$$

The dilaton and 2-form Ramond-Ramond (RR) gauge potential will not be needed and can be found in [28]. We assume without loss of generality ${ }^{4}$

$$
a_{1} \geq a_{2} \geq 0 \text {. }
$$

Depending on the value of the parameters, the geometry can describe a black hole, a naked singularity, a smooth soliton, or a conical singularity [29]. The black hole family of solutions is described by the range $M_{0} \geq\left(a_{1}+a_{2}\right)^{2}$ and has horizons at $g(r)=0$,

$$
r_{ \pm}^{2}=\frac{1}{2}\left(M_{0}-a_{1}^{2}-a_{2}^{2}\right) \pm \frac{1}{2} \sqrt{\left(M_{0}-a_{1}^{2}-a_{2}^{2}\right)^{2}-4 a_{1}^{2} a_{2}^{2}}
$$

\footnotetext{
${ }^{4}$ The simultaneous exchange $a_{1} \rightarrow-a_{1}, \delta_{p} \rightarrow-\delta_{p}, y \rightarrow-y$, and $\psi \rightarrow-\psi$ is a symmetry of the solution. The same is true for $a_{2} \rightarrow-a_{2}, \delta_{p} \rightarrow-\delta_{p}, y \rightarrow-y$, and $\phi \rightarrow-\phi$. So the solutions with $a_{1} a_{2} \leq 0$ are physically equivalent to the solutions with $a_{1} a_{2} \geq 0$. For definiteness we assume the latter.
}

We are particularly interested in the existence of an ergoregion, whose properties were discussed in [29]. The norm of the Killing vector $\partial_{t}$,

$$
\left|\partial_{t}\right|^{2}=-\frac{f-M_{0} c_{p}^{2}}{\sqrt{H_{1} H_{5}}},
$$

becomes spacelike for $f(r)<M_{0} c_{p}^{2}$. This defines a sixdimensional ergoregion, which includes not only the effects of rotation but also of the linear motion of the string. As we mentioned above, and will prove below, the latter does not actually contribute to superradiance. It is therefore more convenient to consider the vector $\zeta=\partial_{t}+\tanh \delta_{p} \partial_{y}$ such that, upon dimensional reduction (so linear momentum becomes charge), its orbits define static asymptotic observers in the five-dimensional black hole geometry, and whose causal character is therefore associated to the rotation ergosphere. Specifically, its norm

$$
|\zeta|^{2}=-\frac{f-M_{0}}{\sqrt{H_{1} H_{5}}}
$$

becomes spacelike for $f(r)<M_{0}$ so a rotational ergosphere appears at $f(r)=M_{0}$.

The Arnowitt-Deser-Misner (ADM) mass $M$, the angular momenta $\left(J_{\phi}, J_{\psi}\right)$, and the gauge charges $\left(Q_{1}, Q_{5}, Q_{p}\right)$ are 
$M=\frac{M_{0}}{2}\left[\cosh \left(2 \delta_{1}\right)+\cosh \left(2 \delta_{5}\right)+\cosh \left(2 \delta_{p}\right)\right]$,

$J_{\phi}=M_{0}\left(a_{2} c_{1} c_{5} c_{p}-a_{1} s_{1} s_{5} s_{p}\right)$,

$J_{\psi}=M_{0}\left(a_{1} c_{1} c_{5} c_{p}-a_{2} s_{1} s_{5} s_{p}\right)$,

$Q_{i}=M_{0} s_{i} c_{i}, \quad i=1,5, p$.

The horizon angular velocities $\Omega_{\phi, \psi}$ along the Cartan angles of $S O(4), \phi$, and $\psi$, are more conveniently written in terms of the Euler left and right rotations in $U(1)_{L} \times$ $U(1)_{R} \subset S U(2)_{L} \times S U(2)_{R} \simeq S O(4)$,

$$
\begin{aligned}
& \Omega_{\phi, \psi}=\frac{1}{2}\left(\Omega_{R} \pm \Omega_{L}\right), \\
& \Omega_{R, L}=\frac{2 \pi}{\beta_{H}} \frac{a_{2} \pm a_{1}}{\left[M_{0}-\left(a_{2} \pm a_{1}\right)^{2}\right]^{1 / 2}} .
\end{aligned}
$$

Following [20], from the surface gravities of the inner and outer horizons $\kappa_{ \pm}$we introduce the temperatures $\beta_{L, R}=$ $1 / T_{L, R}$

$$
\begin{aligned}
\beta_{R, L}= & \frac{2 \pi}{\kappa_{+}} \pm \frac{2 \pi}{\kappa_{-}}, \\
\frac{1}{\kappa_{ \pm}}= & \frac{M_{0}}{2}\left[\frac{c_{1} c_{5} c_{p}+s_{1} s_{5} s_{p}}{\left[M_{0}-\left(a_{2}+a_{1}\right)^{2}\right]^{1 / 2}}\right. \\
& \left. \pm \frac{c_{1} c_{5} c_{p}-s_{1} s_{5} s_{p}}{\left[M_{0}-\left(a_{2}-a_{1}\right)^{2}\right]^{1 / 2}}\right] .
\end{aligned}
$$

Observe that the Hawking temperature of the outer horizon is related to $T_{L, R}$ as in (2.1). Similarly, from the areas of the inner and outer horizons we introduce $S_{L, R}$ such that

$$
\begin{aligned}
S & =S_{L}+S_{R}, \\
S_{R, L} & =\pi M_{0}\left(c_{1} c_{5} c_{p} \mp s_{1} s_{5} s_{p}\right)\left[M_{0}-\left(a_{2} \pm a_{1}\right)^{2}\right]^{1 / 2} .
\end{aligned}
$$

The horizon of the black string is also moving relative to asymptotic observers that follow orbits of $\partial_{t}$. We can compute the linear velocities for both the inner and outer horizons

$$
\begin{aligned}
V_{ \pm}= & \frac{\pi M_{0}}{\beta_{H}}\left[\frac{c_{1} c_{5} s_{p}+s_{1} s_{5} c_{p}}{\left[M_{0}-\left(a_{2}+a_{1}\right)^{2}\right]^{1 / 2}}\right. \\
& \left. \pm \frac{c_{1} c_{5} s_{p}-s_{1} s_{5} c_{p}}{\left[M_{0}-\left(a_{2}-a_{1}\right)^{2}\right]^{1 / 2}}\right]
\end{aligned}
$$

and introduce

$$
V_{R, L}=-\frac{\beta_{H}}{\beta_{R, L}}\left(V_{+} \pm V_{-}\right) .
$$

In terms of these, the velocity of the outer horizon, $V_{+}$, which we also denote as $V_{H}$, is

$$
V_{H}=-\frac{T_{H}}{2}\left(\frac{V_{L}}{T_{L}}+\frac{V_{R}}{T_{R}}\right) .
$$

These velocities become much simpler in the decoupling limit where the D1 and D5 boosts are very large so the system is near supersymmetric, the numbers of anti-D1 and
anti-D5 branes are suppressed, and we can make contact with the dual CFT. In this regime we approximate $c_{1,5} \simeq$ $s_{1,5} \simeq e^{\delta_{1,5}} / 2$ and we find that

$$
V_{L, R} \rightarrow \pm 1 \text {, }
$$

which is microscopically interpreted as the fact that the momentum excitations are chiral and massless. ${ }^{5}$ Observe that in this regime we recover Eq. (2.24), which we had derived from the microscopic two-sector system. The role that the inner horizon plays in defining the microscopic magnitudes associated to the two chiral sectors, emphasized in [20], is very intriguing and not well understood.

During the remainder of this section we will not need to restrict ourselves to this near-supersymmetric regime. But our main interest lies in extremal rotating black hole solutions. These correspond to degenerate horizons, which appear when the two roots $r_{ \pm}$coincide. From (3.4) we identify two possibilities:

(i) The BPS black hole.

Obtained by taking the limit $M_{0} \rightarrow 0, a_{1,2} \rightarrow 0$, keeping the mass, angular momenta, and charges finite, which requires $\delta_{1,5, p} \rightarrow \infty$. In this limit

$$
\begin{gathered}
T_{R} \rightarrow 0, \quad T_{L} \neq 0, \quad S_{R} \rightarrow 0, \quad S_{L} \neq 0, \\
\Omega_{L, R} \rightarrow 0, \quad-V_{R}, V_{L} \rightarrow V_{H} \rightarrow 1 .
\end{gathered}
$$

Also, $J_{\phi}+J_{\psi} \rightarrow 0$, the BPS bound is saturated, the solution is supersymmetric, and the timelike Killing vector that becomes null at the horizon is globally defined, so there is no ergoregion. This is also clear from (3.6). This is the Breckenridge-Myers-PeetVafa (BMPV) black hole.

(ii) The ergo-cold black hole.

Obtained in the limit

$$
M_{0} \rightarrow\left(a_{1}+a_{2}\right)^{2},
$$

in which $T_{H} \rightarrow 0$ but now keeping $\Omega_{R} \neq 0$. Since $M_{0} \neq 0$ the BPS bound is not saturated and supersymmetry is absent. In this limit,

$$
\begin{gathered}
T_{R} \rightarrow 0, \quad T_{L} \neq 0, \quad S_{R} \rightarrow 0, \quad S_{L} \neq 0, \\
\Omega_{L} \rightarrow 0, \quad \Omega_{R} \neq 0, \quad-V_{R} \rightarrow V_{H}, \quad
\end{gathered}
$$

while $V_{L}$ takes no particular value (unless we take the decoupling limit) and the conserved charges $M$, $Q_{i}$, and $J_{\psi, \phi}$ are unconstrained other than by the extremality condition. The horizon does rotate relative to asymptotic observers, and there is an ergosphere, determined by $f(r)=\left(a_{1}+a_{2}\right)^{2}$; see (3.6). Observe that in contrast to the BMPV solution, $J_{\phi}$ and $J_{\psi}$ are independent of each other.

The BMPV black hole has been thoroughly studied, and it will only serve us to emphasize the differences with the

\footnotetext{
${ }^{5} \mathrm{We}$ are taking left velocities and momenta as positive.
} 
ergo-cold black hole, which is our system of choice for the study of superradiance.

\section{B. Absorption cross section and emission rate}

We consider a minimal scalar field, typically a graviton with polarization in the internal $T^{4}$ in the compactification of the IIB theory to six dimensions. The field satisfies the massless Klein-Gordon equation in the general threecharge black string geometry,

$$
\partial_{\mu}\left(\sqrt{-} g g^{\mu \nu} \partial_{\nu} \Phi\right)=0 .
$$

Introducing the ansatz

$$
\Phi=\exp \left[-i \omega t+i p y+i m_{\psi} \psi+i m_{\phi} \phi\right] \chi(\theta) h(r),
$$

and the separation constant $\Lambda$, the wave equation can be separated. The angular equation is

$$
\begin{aligned}
\frac{1}{\sin 2 \theta} \frac{d}{d \theta}\left(\sin 2 \theta \frac{d \chi}{d \theta}\right)+\left[\Lambda-\frac{m_{\psi}^{2}}{\cos ^{2} \theta}-\frac{m_{\phi}^{2}}{\sin ^{2} \theta}\right. \\
\left.+\left(\omega^{2}-p^{2}\right)\left(a_{1}^{2} \sin ^{2} \theta+a_{2}^{2} \cos ^{2} \theta\right)\right] \chi=0 .
\end{aligned}
$$

This angular equation (plus regularity requirements) is a Sturm-Liouville problem, and the solutions are higherdimensional spin-weighted spheroidal harmonics. We can label the corresponding eigenvalues $\Lambda$ with an index $\ell$, $\Lambda(\omega)=\Lambda_{\ell}(\omega)$ and therefore the wave functions form a complete set over the integer $\ell$. In the general case, the problem consists of two coupled second order differential equations: given some boundary conditions, one has to compute simultaneously both values of $\omega$ and $\Lambda$ that satisfy these boundary conditions. However, for vanishing $a_{i}^{2}$ we get the (five-dimensional) flat space result, $\Lambda=$ $\ell(\ell+2)$, and the associated angular functions are given by Jacobi polynomials. For nonzero, but small $\left(\omega^{2}-\right.$ $\left.p^{2}\right) a_{i}^{2}$ we have

$$
\Lambda=\ell(\ell+2)+\mathcal{O}\left(a_{i}^{2}\left(\omega^{2}-p^{2}\right)\right) .
$$

The integer $\ell$ is constrained to be $\ell \geq\left|m_{\phi}\right|+\left|m_{\psi}\right|$, and can only take even (odd) values when $\left|m_{\phi}\right|+\left|m_{\psi}\right|$ is even (odd) [30] - this follows from the fact that the scalar $\ell^{\text {th }}$ wave is in the $(\ell / 2, \ell / 2)$ of $S U(2)_{R} \times S U(2)_{L}$. The angular coordinates $\phi, \psi$ are periodic with period $2 \pi$ so $m_{\phi}, m_{\psi}$ must take integer values. Our waves have positive frequency $\omega>0$.

The radial wave equation can be written in a form that is particularly appropriate to find its solutions. Introduce the new radial coordinate

$$
x=\frac{r^{2}-\frac{1}{2}\left(r_{+}^{2}+r_{-}^{2}\right)}{r_{+}^{2}-r_{-}^{2}},
$$

which maps $r=\left(r_{-}, r_{+}, \infty\right) \leftrightarrow x=(-1 / 2,1 / 2, \infty)$. Introduce also

$$
m_{L, R}=\frac{1}{2}\left(m_{\phi} \mp m_{\psi}\right) .
$$

The radial wave equation is then

$$
\begin{gathered}
\partial_{x}\left[\left(x-\frac{1}{2}\right)\left(x+\frac{1}{2}\right) \partial_{x} h\right]+\frac{1}{4}\left[\left(\omega^{2}-p^{2}\right)\left(r_{+}^{2}-r_{-}^{2}\right) x\right. \\
-(\Lambda-U)] h+\frac{1}{4}\left[\frac{\Sigma_{+}^{2}}{\left(x-\frac{1}{2}\right)}-\frac{\Sigma_{-}^{2}}{\left(x+\frac{1}{2}\right)}\right] h=0,
\end{gathered}
$$

where we defined

$$
\begin{aligned}
\Sigma_{ \pm}= & \frac{\omega}{\kappa_{ \pm}} \mp m_{L} \frac{\Omega_{L}}{\kappa_{+}}-m_{R} \frac{\Omega_{R}}{\kappa_{+}}-p \frac{V_{ \pm}}{\kappa_{+}}, \\
U= & \left(\omega^{2}-p^{2}\right)\left[\frac{1}{2}\left(r_{+}^{2}+r_{-}^{2}\right)+M_{0}\left(s_{1}^{2}+s_{5}^{2}\right)\right] \\
& +\left(\omega c_{p}+p s_{p}\right)^{2} M_{0} .
\end{aligned}
$$

Equation (3.24) was first written (though in a much less compact form) in [31]. For $p=0$ there is no dynamics associated to the sixth direction and (3.24) reduces to the wave equation studied in [20] for the scattering of a neutral scalar off the five-dimensional D1-D5-P black hole.

\section{Near-region wave equation and solution}

In the near region, the term $p^{2}\left(r_{+}^{2}-r_{-}^{2}\right) x$ is suppressed and the radial wave equation reduces to

$$
\begin{aligned}
\partial_{x}[(x & \left.\left.-\frac{1}{2}\right)\left(x+\frac{1}{2}\right) \partial_{x} h\right] \\
& +\frac{1}{4}\left[-(\Lambda-U)+\frac{\Sigma_{+}^{2}}{\left(x-\frac{1}{2}\right)}-\frac{\Sigma_{-}^{2}}{\left(x+\frac{1}{2}\right)}\right] h=0 .
\end{aligned}
$$

To find the analytical solution of this equation, define the new radial coordinate,

$$
\begin{aligned}
& z=x+\frac{1}{2}, \\
& r=\left(r_{-}, r_{+}, \infty\right) \leftrightarrow x=(-1 / 2,1 / 2, \infty) \leftrightarrow z=(0,1, \infty),
\end{aligned}
$$

and introduce the new wave function

$$
h=z^{-i(1 / 2) \Sigma_{-}}(z-1)^{-i(1 / 2) \Sigma_{+}} F .
$$

The near-region radial wave equation can then be written as

$$
\begin{aligned}
& z(1-z) \partial_{z}^{2} F+\left[\left(1-i \Sigma_{-}\right)-\left[2-i\left(\Sigma_{+}+\Sigma_{-}\right)\right] z\right] \partial_{z} F \\
& \quad+\left[i \frac{1}{4}\left(\Sigma_{+}+\Sigma_{-}\right)\left[2-i\left(\Sigma_{+}+\Sigma_{-}\right)\right]+(\Lambda-U)\right] F=0,
\end{aligned}
$$

which is a standard hypergeometric equation [32], $z(1-$ z) $\partial_{z}^{2} F+[c-(a+b+1) z] \partial_{z} F-a b F=0$, with 


$$
\begin{gathered}
a=\xi-\frac{i}{2}\left(\Sigma_{+}+\Sigma_{-}\right), \quad b=1-\xi-\frac{i}{2}\left(\Sigma_{+}+\Sigma_{-}\right), \\
c=1-i \Sigma_{-},
\end{gathered}
$$

where we defined

$$
\xi=\frac{1}{2}(1+\sqrt{1+\Lambda-U}) .
$$

Its most general solution in the neighborhood of $z=1$ (i.e., $\left.r=r_{+}\right)$is $A_{H}^{\text {in }} z^{-b} F\left(b, b-c+1, a+b-c+1, \frac{z-1}{z}\right)+$ $A_{H}^{\text {out }} z^{a-c}(z-1)^{c-a-b} F\left(c-a, 1-a, c-a-b+1, \frac{z-1}{z}\right)$.

Using (3.28), one finds that the solution of the near-region equation is

$$
\begin{aligned}
h= & A_{H}^{\text {in }}\left(x-\frac{1}{2}\right)^{-i(1 / 2) \Sigma_{+}}\left(x+\frac{1}{2}\right)^{-\xi+i(1 / 2) \Sigma_{+}} F\left(b, b-c+1, a+b-c+1, \frac{x-\frac{1}{2}}{x+\frac{1}{2}}\right) \\
& +A_{H}^{\text {out }}\left(x-\frac{1}{2}\right)^{+i(1 / 2) \Sigma_{+}}\left(x+\frac{1}{2}\right)^{-\xi} F\left(c-a, 1-a, c-a-b+1, \frac{x-\frac{1}{2}}{x+\frac{1}{2}}\right) .
\end{aligned}
$$

The first term represents an ingoing wave at the horizon $x=\frac{1}{2}$, while the second term represents an outgoing wave at the horizon. The computation of the absorption cross section is a classical problem where outgoing waves at the horizon are forbidden, so we set $A_{H}^{\text {out }}=0$. Furthermore, we need the large $r, x \rightarrow \infty$ behavior of the ingoing near-region solution. We use the $z \rightarrow 1-z$ transformation law for the hypergeometric function [32],

$$
\begin{aligned}
F\left(b, b-c+1, a+b-c+1, \frac{x-\frac{1}{2}}{x+\frac{1}{2}}\right)= & \frac{\Gamma(a+b-c+1) \Gamma(a-b)}{\Gamma(a-c+1) \Gamma(a)} F\left(b, b-c+1,-a+b+1, \frac{1}{x+\frac{1}{2}}\right) \\
& +\left(x+\frac{1}{2}\right)^{a-b} \frac{\Gamma(a+b-c+1) \Gamma(-a+b)}{\Gamma(b) \Gamma(b-c+1)} F\left(a-c+1, a, a-b+1, \frac{1}{x+\frac{1}{2}}\right),
\end{aligned}
$$

the property $F(a, b, c, 0)=1$, and $x \pm \frac{1}{2} \sim x$. The large $x$ behavior of the ingoing near-horizon solution is then

$$
h \sim A_{H}^{\text {in }}\left[\frac{\Gamma\left[1-i \Sigma_{+}\right] \Gamma[1-2 \xi]}{\Gamma\left[1-\xi-i \frac{1}{2}\left(\Sigma_{+}-\Sigma_{-}\right)\right] \Gamma\left[1-\xi-i \frac{1}{2}\left(\Sigma_{+}+\Sigma_{-}\right)\right]} x^{-\xi}+\frac{\Gamma\left[1-i \Sigma_{+}\right] \Gamma[1-2 \xi]}{\Gamma\left[\xi-i \frac{1}{2}\left(\Sigma_{+}-\Sigma_{-}\right)\right] \Gamma\left[\xi-i \frac{1}{2}\left(\Sigma_{+}+\Sigma_{-}\right)\right.} x^{\xi-1}\right] .
$$

\section{Far-region wave equation and solution}

In the far region, the terms $\left(x \pm \frac{1}{2}\right)^{-1}$ are suppressed, and $x \pm \frac{1}{2} \sim x$. The radial wave equation can be written as

$$
\partial_{x}^{2}(x h)+\left[\frac{\left(\omega^{2}-p^{2}\right)\left(r_{+}^{2}-r_{-}^{2}\right)}{4 x}-\frac{\Lambda-U}{4 x^{2}}\right](x h)=0 .
$$

The most general solution of this equation is a linear combination of Bessel functions [32],

$$
h=x^{-1 / 2}\left[A_{\infty}^{+} J_{2 \xi-1}\left(\mu x^{1 / 2}\right)+A_{\infty}^{-} J_{1-2 \xi}\left(\mu x^{1 / 2}\right)\right],
$$

where $\xi$ was defined in (3.31) and

$$
\mu=\left[\left(\omega^{2}-p^{2}\right)\left(r_{+}^{2}-r_{-}^{2}\right)\right]^{1 / 2} .
$$

We want to study the scattering process so we require real $\mu$ i.e., $\omega>|p|$. Using the asymptotic properties of the Bessel function [32], we find that for small $\mu x^{1 / 2}$ the farregion solution has the behavior

$$
h \sim A_{\infty}^{+} \frac{(\mu / 2)^{2 \xi-1}}{\Gamma(2 \xi)} x^{\xi-1}+A_{\infty}^{-} \frac{(\mu / 2)^{1-2 \xi}}{\Gamma(2-2 \xi)} x^{-\xi},
$$

while for large $\mu x^{1 / 2}$ it reduces to

$$
\begin{aligned}
h \sim & \frac{1}{2} \sqrt{\frac{2}{\pi \mu}} x^{-3 / 4}\left\{\left[A_{\infty}^{+} e^{-i \pi(-\xi+1 / 4)}+A_{\infty}^{-} e^{-i \pi(\xi-3 / 4)}\right]\right. \\
& \left.\times e^{-i \mu \sqrt{x}}+\left[A_{\infty}^{+} e^{i \pi(-\xi+1 / 4)}+A_{\infty}^{-} e^{i \pi(\xi-3 / 4)}\right] e^{i \mu \sqrt{x}}\right\} .
\end{aligned}
$$

The first term represents an incoming wave while the second term describes an outgoing solution.

\section{Matching the near-region and the far-region solutions}

There is an intermediate region for $x$ where the approximations in both the near and far regions can be simultaneously satisfied. In this overlapping region we can match the large $x$ behavior of the near-region solution to the small $x$ behavior of the far-region solution. This allows to fix the amplitude ratios. Matching (3.34) with (3.38) yields then 


$$
\begin{aligned}
& \frac{A_{H}^{\text {in }}}{A_{\infty}^{+}}=\left(\frac{\mu}{2}\right)^{2 \xi-1} \frac{\Gamma\left[\xi-i \frac{1}{2}\left(\Sigma_{+}-\Sigma_{-}\right)\right] \Gamma\left[\xi-i \frac{1}{2}\left(\Sigma_{+}+\Sigma_{-}\right)\right]}{\Gamma(2 \xi) \Gamma(2 \xi-1) \Gamma\left[1-i \Sigma_{+}\right]}, \\
& \frac{A_{\infty}^{-}}{A_{\infty}^{+}}=\left(\frac{\mu}{2}\right)^{2(2 \xi-1)} \frac{\Gamma(2-2 \xi) \Gamma(1-2 \xi)}{\Gamma(2 \xi) \Gamma(2 \xi-1)} \frac{\Gamma\left[\xi-i \frac{1}{2}\left(\Sigma_{+}-\Sigma_{-}\right)\right] \Gamma\left[\xi-i \frac{1}{2}\left(\Sigma_{+}+\Sigma_{-}\right)\right]}{\Gamma\left[1-\xi-i \frac{1}{2}\left(\Sigma_{+}-\Sigma_{-}\right)\right] \Gamma\left[1-\xi-i \frac{1}{2}\left(\Sigma_{+}+\Sigma_{-}\right)\right]} .
\end{aligned}
$$

The first relation will be needed to compute the absorption cross section. In the second relation we note the presence of the factor $\mu^{2 \xi-1}$, where $\mu$ is defined in (3.37). We want $\xi \in \mathbb{R}$ which implies $2 \xi-1>0$. Therefore, for $\mu \ll 1$, i.e., for low frequency scattering or for nearsupersymmetric solutions (decoupling limit), one has $\left|A_{\infty}^{-}\right| \ll\left|A_{\infty}^{+}\right|$. This regime allows to considerably simplify (3.39).

\section{Absorption cross section, Hawking and superradiant emission rate} is

The radial flux associated with our radial wave equation

$$
\mathcal{F}=\frac{1}{2 i}\left(h^{*} \frac{g(r)}{r} \partial_{r} h-h \frac{g(r)}{r} \partial_{r} h^{*}\right) .
$$

The incoming flux from infinity $\mathcal{F}_{\text {in }}$ is computed using (3.39). Near the decoupling regime $\left|A_{\infty}^{-}\right| \ll\left|A_{\infty}^{+}\right|$, this yields

$$
\mathcal{F}_{\text {in }}=-\frac{r_{+}^{2}-r_{-}^{2}}{2 \pi}\left|A_{\infty}^{+}\right|^{2}
$$

where the minus sign signals incoming flux. On the other hand, use of the ingoing contribution of (3.32) yields for the absorbed flux at the horizon,

$$
\mathcal{F}_{\text {abs }}=-\Sigma_{+}\left(r_{+}^{2}-r_{-}^{2}\right)\left|A_{H}^{\text {in }}\right|^{2} .
$$

The absorption probability is the ratio of the above fluxes,

$$
1-\left|S_{\ell}\right|^{2}=\frac{\mathcal{F}_{\text {abs }}}{\mathcal{F}_{\text {in }}},
$$

and the absorption cross section of the $\ell^{\text {th }}$ partial wave is

$$
\sigma_{\ell, p, m_{R, L}}=\frac{4 \pi}{\omega^{3}}(\ell+1)^{2}\left(1-\left|S_{\ell}\right|^{2}\right)
$$

In general, the factor multiplying the absorption probability depends on the spacetime dimension through the codimension of the absorbing object (see, e.g., [11]). So for a six-dimensional black string we use the same factor as for a five-dimensional black hole. Collecting the results, the absorption cross section is

$$
\begin{aligned}
\sigma_{\ell, p, m_{R, L}}= & \frac{4 \pi(\ell+1)^{2}}{\omega^{3}} \beta_{H} \varpi\left[\frac{1}{4}\left(\omega^{2}-p^{2}\right)\left(r_{+}^{2}-r_{-}^{2}\right)\right]^{2 \xi-1} \\
& \times\left|\frac{\Gamma\left(\xi-i \frac{\beta_{L} \varpi_{L}}{2 \pi}\right) \Gamma\left(\xi-i \frac{\beta_{R} \varpi_{R}}{2 \pi}\right)}{\Gamma(2 \xi) \Gamma(2 \xi-1) \Gamma\left(1-i \frac{\beta_{H} \varpi}{2 \pi}\right)}\right|^{2}
\end{aligned}
$$

where we defined

$$
\begin{aligned}
\varpi & =\omega-p V_{H}-m_{L} \Omega_{L}-m_{R} \Omega_{R}, \\
\varpi_{L, R} & =\frac{1}{2}\left(\omega+p V_{L, R}\right)-m_{L, R} \Omega_{L, R} \frac{\beta_{H}}{\beta_{L, R}} .
\end{aligned}
$$

Observe in the latter equation the presence of $\Omega_{L, R} \frac{\beta_{H}}{\beta_{L, R}}$, which correspond to the chemical potentials $\mu_{L, R}$ of the microscopic two-sector system (2.9).

The matching (3.40) was performed in the low frequency regime of waves with wavelength much larger than the typical size of the black hole. This is actually the regime of relevance when comparing to the microscopic dual, in which the excitations near the horizon are (almost) decoupled from the asymptotic region, and we only allow a little leakage of energy between the two regions. The latter corresponds to coupling the dual CFT to a bulk scalar. Using (3.21) and (3.25) this is the range of parameters where

$$
U \ll \Lambda \simeq \ell(\ell+2) \Rightarrow \xi \simeq \frac{\ell+2}{2} .
$$

In particular, since $\xi$ is integer or half-integer, the following relations [32]

$$
\begin{aligned}
|\Gamma(n-i z)|^{2} & =\Gamma(n-i z) \Gamma(n+i z), \\
\Gamma(n \pm i z) & =\Gamma(1 \pm i z) \prod_{j=1}^{n-1}\left(j^{2}+z^{2}\right), \\
|\Gamma(1-i z)|^{2} & =\frac{\pi z}{\sinh (\pi z)}, \\
\left|\Gamma\left(\frac{1}{2}-i z\right)\right|^{2} & =\frac{\pi}{\cosh (\pi z)},
\end{aligned}
$$

are useful. Thus we can rewrite (3.46) as 


$$
\begin{aligned}
\sigma_{\ell, p, m_{R, L}}= & \frac{8 \pi}{(\ell !)^{4}} \frac{1}{\omega^{3}}\left(\left(\omega^{2}-p^{2}\right) \frac{\mathcal{A}_{H}^{(5)}}{4 \pi \beta_{H}}\right)^{\ell+1} \sinh \left(\frac{\beta_{H} \varpi}{2}\right) \\
& \times\left|\Gamma\left(\frac{\ell+2}{2}+i \frac{\beta_{L} \varpi_{L}}{2 \pi}\right) \Gamma\left(\frac{\ell+2}{2}+i \frac{\beta_{R} \varpi_{R}}{2 \pi}\right)\right|^{2},
\end{aligned}
$$

where we have used $r_{+}^{2}-r_{-}^{2}=\mathcal{A}_{H}^{(5)} /\left(4 G_{5} \beta_{H}\right)$ with $\mathcal{A}_{H}^{(5)}$ the area of the five-dimensional black hole, and in our units $G_{5}=\pi / 4$.
By detailed balance, the decay rate is the absorption cross section divided by the thermal Bose-Einstein occupation number,

$$
\Gamma_{\ell, p, m_{R, L}}=\frac{\sigma_{\ell, p, m_{R, L}}}{e^{\beta_{H} \varpi}-1} .
$$

Use of (3.49) also allows to write the decay rate in terms of thermal factors. We have to distinguish the cases of even and odd angular quantum number $\ell$. For even $\ell$, (3.46), (3.49), and (3.51) give the decay rate,

$$
\begin{aligned}
\text { Even } \ell: \Gamma_{\ell, p, m_{R, L}}= & \frac{4 \pi}{(\ell !)^{4}}\left[\left(\omega^{2}-p^{2}\right) \frac{\mathcal{A}_{H}^{(5)}}{4 \pi}\right]^{\ell+1} \frac{\varpi_{L} \varpi_{R}}{\omega^{3}} \frac{\beta_{L} \beta_{R}}{\beta_{H}}\left(e^{\beta_{L} \varpi_{L}}-1\right)^{-1}\left(e^{\beta_{R} \varpi_{R}}-1\right)^{-1} \\
& \times \prod_{j=1}^{\ell / 2}\left[j^{2}+\left(\frac{\beta_{L} \varpi_{L}}{2 \pi}\right)^{2}\right]\left[\left(\frac{j}{\beta_{H}}\right)^{2}+\left(\frac{\beta_{R} \varpi_{R}}{2 \pi \beta_{H}}\right)^{2}\right] .
\end{aligned}
$$

For odd $\ell$, the decay rate is

$$
\begin{aligned}
\text { Odd } \ell: \Gamma_{\ell, p, m_{R, L}}= & \frac{2(2 \pi)^{3}}{(\ell !)^{4}}\left[\left(\omega^{2}-p^{2}\right) \frac{\mathcal{A}_{H}^{(5)}}{4 \pi}\right]^{\ell+1} \frac{1}{\omega^{3}}\left(e^{\beta_{L} \varpi_{L}}+1\right)^{-1}\left(e^{\beta_{R} \varpi_{R}}+1\right)^{-1} \\
& \times \prod_{j=1}^{(\ell+1) / 2}\left[\left(j-\frac{1}{2}\right)^{2}+\left(\frac{\beta_{L} \varpi_{L}}{2 \pi}\right)^{2}\right]\left[\left(\frac{2 j-1}{2 \beta_{H}}\right)^{2}+\left(\frac{\beta_{R} \varpi_{R}}{2 \pi \beta_{H}}\right)^{2}\right] .
\end{aligned}
$$

As observed in [9], for even $\ell$ there appear left and right bosonic thermal factors (3.52) while for odd $\ell$ they are fermionic thermal factors. This is already a hint of the microscopic degrees of freedom responsible for the radiation-taking into account that the bosonic factors can emerge as effective ones from even numbers of fermions $[10,33]$.

\section{Superradiant emission rate from the ergo-cold black hole}

These emission rates contain effects of Hawking radiation as well as superradiance. As explained in the introduction, in order to eliminate the former we take an extremal, zero-temperature limit, while at the same time we want to preserve the superradiant ergoregion.

In the case of the supersymmetric BMPV black hole, neither thermal nor superradiant emissions are present. In the limit to this solution

$$
\lim _{\beta_{R} \rightarrow \infty} \varpi_{R}=\frac{\omega}{2}>0
$$

and the positivity of $\varpi_{R}$ implies that, in (3.52) and (3.53), the right thermal factor $\left(e^{\beta_{R} \varpi_{R}} \pm 1\right)^{-1} \rightarrow 0$, so $\Gamma_{\ell, p, m_{R, L}}=$ 0 . This is as it should be, since this is a BPS state. The absorption cross section is positive for any quantum numbers of the wave, so stimulated emission cannot occur either.

The ergo-cold black hole is obtained in the limit in which $\beta_{R} \rightarrow \infty$ while $\Omega_{R}$ remains finite. In this case

$$
\begin{aligned}
& \lim _{\beta_{R} \rightarrow \infty} \varpi_{L}=\frac{1}{2}\left(\omega+p V_{L}\right)-m_{L} \frac{\pi\left(a_{2}-a_{1}\right) \sqrt{a_{1} \overline{a_{2}}}}{\beta_{L}}, \\
& \lim _{\beta_{R} \rightarrow \infty} \varpi_{R}=\frac{1}{2}\left(\omega-p V_{H}-m_{R} \Omega_{R}\right) .
\end{aligned}
$$

Now $\varpi_{R}$ can take negative values, so the decay rates do not vanish for all modes but contain a factor of a step function

$$
\lim _{\beta_{R} \rightarrow \infty}\left(e^{\beta_{R} \varpi_{R}} \pm 1\right)^{-1}=\mp \Theta\left(-\varpi_{R}\right),
$$

so the emission decay rate is

$$
\begin{aligned}
\text { Even } \ell: \Gamma_{\ell, p, m_{R, L}}= & \Theta\left(-\varpi_{R}\right) \frac{8 \pi^{2}}{(\ell !)^{4}}\left[\left(\omega^{2}-p^{2}\right) \frac{\mathcal{A}_{H}^{(5)}}{4 \pi^{2}}\right]^{\ell+1} \\
& \times \frac{\beta_{L} \varpi_{L}\left|\varpi_{R}\right|^{\ell+1}}{\omega^{3}\left(e^{\beta_{L} \varpi_{L}}-1\right)} \prod_{j=1}^{\ell / 2}\left[j^{2}+\left(\frac{\beta_{L} \varpi_{L}}{2 \pi}\right)^{2}\right], \\
\operatorname{Odd} \ell: \Gamma_{\ell, p, m_{R, L}}= & \Theta\left(-\varpi_{R}\right) \frac{2(2 \pi)^{3}}{(\ell !)^{4}}\left[\left(\omega^{2}-p^{2}\right) \frac{\mathcal{A}_{H}^{(5)}}{4 \pi^{2}}\right]^{\ell+1} \\
& \times \frac{\left|\varpi_{R}\right|^{\ell+1}}{\omega^{3}\left(e^{\left.\beta_{L} \varpi_{L}+1\right)}\right.} \times \prod_{j=1}^{(\ell+1) / 2}\left[\left(j-\frac{1}{2}\right)^{2}\right. \\
& \left.+\left(\frac{\beta_{L} \varpi_{L}}{2 \pi}\right)^{2}\right] .
\end{aligned}
$$

Thus we have derived the superradiant bound (2.21). The ergo-cold black hole can only emit modes that satisfy $\varpi_{R}<0$. The absorption cross section is positive or negative depending on whether $\varpi_{R}$ is positive or negative, so 
when $\varpi_{R}<0$, and only then, superradiant amplification occurs.

We can also see that there cannot be any spinless, pure momentum superradiance. An oscillating wave near infinity must have $\omega>|p|$. Technically, this follows from the reality requirement of quantities like (3.37) or (3.57). Physically, $\omega^{2}-p^{2}>0$ for a wave propagating in the asymptotically flat region. According to (3.55), spinless superradiant modes require $\omega<p V_{H}$. But (3.12) gives at extremality $V_{H}=\frac{c_{1} c_{5} s_{p}+s_{1} s_{5} c_{p}}{c_{1} c_{5} c_{p}+s_{1} s_{5} s_{p}}$ so $\left|V_{H}\right| \leq 1$ and $\left|p V_{H}\right| \leq$ $|p|$. Then, none of these superradiant momentum modes can exist as propagating waves at infinity: if emitted by the black hole, they will be reflected back to it before getting to the asymptotic region. This is a general feature present in black string backgrounds [34,35].

\section{MICROSCOPIC DESCRIPTION}

\section{A. The dual CFT state}

The CFT state dual to the ergo-cold black hole is most easily identified by analyzing the solution in the decoupling limit. This is a low-energy limit, keeping the energies finite in string units, which is obtained taking $\alpha^{\prime} \rightarrow 0$ and $\delta_{1,5} \rightarrow \infty$ while keeping $r\left(\alpha^{\prime}\right)^{-1}, M\left(\alpha^{\prime}\right)^{-2}, a_{1,2}\left(\alpha^{\prime}\right)^{-1}$, and $Q_{1,5}\left(\alpha^{\prime}\right)^{-1}$ fixed. For the general black hole geometry, this has been shown to result in a twisted fibration of $S^{3}$ over the Bañados-Teitelboim-Zanelli (BTZ) black hole [21]. The CFT states dual to the extremal black holes we have been studying can be identified using the map introduced in [36]. This yields the R-charges $(j, \bar{j})$ and conformal dimensions $(h, \bar{h})$ of the CFT state in terms of parameters of the supergravity solution. Introducing the $\mathrm{AdS}_{3}$ curvature radius $\ell_{3}$, BTZ black hole mass $M_{3}$,

$$
\begin{aligned}
\ell_{3}^{2} & =\sqrt{Q_{1} Q_{5}}, \\
M_{3} & =\frac{R^{2}}{\ell^{4}}\left[\left(M-a_{1}^{2}-a_{2}^{2}\right)\left(c_{p}^{2}+s_{p}^{2}\right)+4 a_{1} a_{2} s_{p} c_{p}\right],
\end{aligned}
$$

and central charge $c=3 \ell_{3} / 2$, the following values are obtained for the two extremal rotating black holes:

(i) BMPV black hole:

$$
\begin{gathered}
j=\frac{c}{6} \frac{R}{\ell_{3}^{4}} J_{L}, \quad h=\frac{c}{24}\left(1+2 M_{3}+\frac{4 R^{2}}{\ell_{3}^{8}} J_{L}^{2}\right), \\
\bar{j}=0, \quad \bar{h}=\frac{c}{24} .
\end{gathered}
$$

(ii) Ergo-cold black hole:

$$
\begin{gathered}
j=\frac{c}{6} \frac{R}{\ell_{3}^{4}} J_{L}, \quad h=\frac{c}{24}\left(1+2 M_{3}+\frac{4 R^{2}}{\ell_{3}^{8}} J_{L}^{2}\right), \\
\bar{j}=\frac{c}{6} \frac{R}{\ell_{3}^{4}} J_{R}, \quad \bar{h}=\frac{c}{24}\left(1+\frac{4 R^{2}}{\ell_{3}^{8}} J_{R}^{2}\right) .
\end{gathered}
$$

To interpret these results we note that the conformal dimensions receive contributions of three kinds,

$$
h=h_{0}+l_{0}+\frac{6 j^{2}}{c}, \quad \bar{h}=\bar{h}_{0}+\bar{l}_{0}+\frac{6 \bar{j}^{2}}{c} .
$$

Here $\left(h_{0}, \bar{h}_{0}\right)=(c / 24, c / 24)$ correspond to the energy of the Ramond ground state. On top of this, the left sector has in both cases an excitation energy given by the Virasoro level $l_{0}=\ell_{3} M_{3} / 8$ : this is the energy of its thermal excitations, which give the system a Cardy entropy

$$
S_{L}=2 \pi \sqrt{c l_{0} / 6}
$$

Additionally, the left sector contains some polarized fermions, which yield a charge $j$. The Kac-Moody level of the superconformal current algebra is $k=2 c / 3$. The Sugawara stress-energy tensor of the $S U(2)$ current then yields an energy $(2 j)^{2} / k=6 j^{2} / c$, where $2 j$ appears since we are normalizing $j$ to be half-integer quantized.

The right sector in both black holes is at zero level, $\bar{l}_{0}=$ 0 , so they are at vanishing temperature. But there is a crucial difference between the two states: whereas in the BMPV black hole this sector is in a Ramond ground state, in the ergo-cold black hole it is filled with polarized fermions, giving charge $\bar{j}$ and additional energy $6 \bar{j}^{2} / c$ that lifts the system above the BPS state. This is the microscopic picture that we are advocating for this black hole.

\section{B. Emission rate and absorption cross section}

A coupling of the schematic form

$$
S_{\text {int }} \propto \int d t d x \partial^{\ell} \Phi(t, x, \vec{x}=0) \mathcal{O}(t, x)
$$

( $t, x$ are world-sheet coordinates and $\vec{x}$ are directions transverse to the string) describes the interaction of the $\ell^{\text {th }}$ partial wave of the bulk scalar $\Phi$ with an operator $\mathcal{O}(t, x)$ of the CFT of conformal dimension $(1+\ell / 2,1+$ $\ell / 2$ ). We build the latter out of a pair of bosons $\partial_{ \pm} X$, and $\ell$ pairs of left and right fermions $\psi_{L} \bar{\psi}_{R}$. This coupling gives a decay rate of the CFT into a scalar mode with quantum numbers $\omega, \ell, p, m_{R, L}$, of the form

$$
\Gamma_{\ell, p, m_{R, L}}(\omega)=\mathcal{V} \int d x^{+} d x^{-} e^{-i \varpi_{R} x^{-}-i \varpi_{L} x^{+}} \mathcal{G}(t-i \varepsilon, x),
$$

where $x^{ \pm}=t \pm x$, the Green's function is

$$
\mathcal{G}(t, x)=\left\langle\mathcal{O}^{\dagger}(t, x) \mathcal{O}(0)\right\rangle,
$$

with the $i \varepsilon$ prescription in (4.7) corresponding to emission, $\mathcal{V}$ is a factor from the interaction vertex to be discussed below, and

$$
\varpi_{L, R}=\frac{1}{2}(\omega \pm p)-m_{L, R} \mu_{L, R}
$$

account for the presence of left and right sectors with chiral momenta $(\omega \pm p) / 2$ and chemical potentials $\mu_{L, R}$ for the 
$\mathrm{R}$-charges $m_{R, L}$, given by (2.9). These $\varpi_{L, R}$ coincide with those defined for supergravity in (3.47) if we take the decoupling limit in which $V_{L, R} \rightarrow \pm 1$.

\section{Superradiant bound}

We can easily derive from these formulas the bound on decay frequencies for the CFT state dual to the black hole (4.3). For this state, the left sector is at temperature $T_{L}$ so the left-chirality operator $\mathcal{O}_{L}\left(x^{+}\right)$gives in (4.8) a thermal two-point function periodic in imaginary time,

$$
\left\langle\mathcal{O}_{L}^{\dagger}\left(x^{+}\right) \mathcal{O}_{L}(0)\right\rangle_{T_{L}} \sim\left(\frac{\pi T_{L}}{\sinh \left(\pi T_{L} x^{+}\right)}\right)^{2+\ell}
$$

The right sector is at zero temperature, and so the boson gives the two-point function $\partial_{-} X\left(x^{-}\right) \partial_{-} X(0) \sim 1 /\left(x^{-}\right)^{2}$ and each fermion gives $\psi\left(x^{-}\right) \psi(0) \sim 1 / x^{-}$, so

$$
\left\langle\mathcal{O}_{R}^{\dagger}\left(x^{-}\right) \mathcal{O}_{R}(0)\right\rangle_{0} \sim\left(\frac{1}{x^{-}}\right)^{2+\ell},
$$

and the integration over the right sector in the decay rate (4.7) gives a factor

$$
\int d x^{-} e^{-i \varpi_{R} x^{-}}\left(\frac{1}{x^{-}-i \varepsilon}\right)^{2+\ell}
$$

This contour integral vanishes for $\varpi_{R}>0$, so

$$
\Gamma_{\ell, p, m_{R, L}}(\omega) \propto \Theta\left(-\varpi_{R}\right) .
$$

This bound on frequencies coincides with the one we obtained from the supergravity side (3.55) and (3.57) in the extremal limit where $\mu_{R} \rightarrow \Omega_{R} / 2$ (2.10), and in the decoupling limit in which $V_{H} \rightarrow 1$. We feel, nevertheless, that the microscopic derivation we gave in Sec. II is physically more transparent.

\section{Absorption cross section: general case}

It is actually possible to compute the absorption cross section for the more general case where both sectors are at temperatures $T_{L}$ and $T_{R}$ so we can compare it with the general results we obtained from the supergravity side. We follow $[9,22,23]$ but discuss the general case with nonvanishing $\mu_{L, R}$ and $p$. The Green's function (4.8) now has thermal correlation functions from both sectors,

$$
\begin{aligned}
\mathcal{G}(t, x)= & (-1)^{\ell} C_{\mathcal{O}}\left(\frac{\pi T_{L}}{\sinh \left(\pi T_{L} x^{+}\right)}\right)^{2+\ell} \\
& \times\left(\frac{\pi T_{R}}{\sinh \left(\pi T_{R} x^{-}\right)}\right)^{2+\ell},
\end{aligned}
$$

where we include a factor $C_{\mathcal{O}}=C_{\mathcal{O}_{+}} C_{\mathcal{O}_{-}}$that accounts for the normalization factors of the operators, and which we will discuss below jointly with the vertex factor $\mathcal{V}$. The CFT absorption cross section is the difference between absorption and emission rates divided by the flux $\mathcal{F}$. Then,

$$
\begin{aligned}
\sigma_{\ell, p, m_{R, L}}^{\mathrm{CFT}} & =\frac{\mathcal{V}}{\mathcal{F}} \int d x^{+} d x^{-} e^{-i\left(\varpi_{R} x^{-}+\varpi_{L} x^{+}\right)}[G(t-i \epsilon, x)-G(t+i \epsilon, x)] \\
& =\frac{C_{\mathcal{O}} \mathcal{V}}{\mathcal{F}} \frac{\left(2 \pi T_{L}\right)^{1+\ell}\left(2 \pi T_{R}\right)^{1+\ell}}{\Gamma(2+\ell)^{2}} \times \sinh \left(\frac{\varpi}{2 T_{H}}\right)\left|\Gamma\left(\frac{\ell+2}{2}+i \frac{\varpi_{L}}{2 \pi T_{L}}\right) \Gamma\left(\frac{\ell+2}{2}+i \frac{\varpi_{R}}{2 \pi T_{R}}\right)\right|^{2} .
\end{aligned}
$$

Here $\varpi$ is exactly the same quantity that we introduced in the supergravity analysis in (3.47), with $V_{H}$ given in terms of $T_{L, R}$ as in (2.24).

In order to find the factor $C_{\mathcal{O}} \mathcal{V}$, we first determine it for s-wave $(\ell=0)$ absorption by the six-dimensional string (see [23] for more details). The minimally coupled scalar $\Phi$ comes from an internally polarized graviton, say $h_{67}$, so for $\ell=0$ the operator $\mathcal{O}$ is $\partial_{-} X_{A}^{6} \partial_{+} X_{A}^{7}$, where the index $A=1, \ldots N_{1} N_{5}=Q_{1} Q_{5} / R$ refers to the twist sector of the orbifold CFT. Canonical normalization of the scalar field yields a factor $16 \pi G_{6}=8 \pi^{3} R$ in our units where $G_{5}=$ $\pi / 4$, times a factor of $2^{2}$ for the doubling due to $h_{67}=h_{76}$. On the other hand, we find a conventional factor $1 / 16 \pi^{2}$ from the two-point function of $\partial_{-} X \partial_{+} X$, and since we are in the maximally twisted sector we must sum over all values of $A$. This determines

$$
\left(C_{\mathcal{O}} \mathcal{V}\right)_{\ell=0}=4 \times 8 \pi^{3} R \frac{Q_{1} Q_{5}}{16 \pi^{2} R}=2 \pi Q_{1} Q_{5}
$$

When $\ell>0$ the precise form of the vertex factor requires an explicit derivation of the interaction vertex from string theory. We shall not pursue this, but instead follow [10] to find heuristically its dependence on all black hole parameters and all wave quantum numbers except for $\ell$. The $Q_{1} Q_{5}$ flavors of open string fermions in the long string, combined along the boundary of the disk diagram, yield a factor $\left(Q_{1} Q_{5}\right)^{\ell}$ for the $\ell$ fermion pairs entering the interaction. We must also divide it by $(\ell !)^{2}$ to account for the fact that we are overcounting possibilities since the $\ell$ fermions in each sector are indistinguishable. Additionally, the vertex must at least contain the $\ell$ factors of momentum from the derivatives in it. Each of the left and right fermions contribute, respectively, with $(\omega \mp p) / 2$ to this factor, yielding a total

$$
C_{\mathcal{O}} \mathcal{V}=2 \pi Q_{1} Q_{5}\left[\frac{1}{4}\left(\omega^{2}-p^{2}\right)\right]^{\ell} \frac{\left(Q_{1} Q_{5}\right)^{\ell}}{(\ell !)^{2}} \hat{\mathcal{V}}_{\ell}
$$


where there remains an undetermined $\ell$-dependent factor $\hat{V}_{\ell}$, such that $\hat{V}_{\ell=0}=1$.

The flux $\mathcal{F}$ measures the frequency or energy flow per unit cross section. For a scalar of frequency $\omega$ and vanishing momentum $p=0$ the canonically normalized flux of the incident field is $\mathcal{F}=\omega$. However, if it has momentum $p$, then in the frame of the string the frequency is increased by a Lorentz factor $\left(1-p^{2} / \omega^{2}\right)^{-1 / 2}$, while the cross section is Lorentz contracted by $\left(1-p^{2} / \omega^{2}\right)^{1 / 2}$. Therefore, in (4.15) the flux is

$$
\mathcal{F}=\frac{\omega}{1-p^{2} / \omega^{2}} .
$$

The final result is then

$\sigma_{\ell, p, m_{R, L}}^{\mathrm{CFT}}=\frac{8 \pi \hat{\mathcal{V}}_{\ell}}{(\ell !(\ell+1) !)^{2}} \frac{1}{\omega^{3}}\left(\frac{\omega^{2}-p^{2}}{4}\right)^{\ell+1}\left(4 \pi^{2} T_{L} T_{R} Q_{1} Q_{5}\right)^{\ell+1} \sinh \left(\frac{\varpi}{2 T_{H}}\right) \times\left|\Gamma\left(\frac{\ell+2}{2}+i \frac{\varpi_{L}}{2 \pi T_{L}}\right) \Gamma\left(\frac{\ell+2}{2}+i \frac{\varpi_{R}}{2 \pi T_{R}}\right)\right|^{2}$

In order to compare this with the result (3.50) from supergravity, we must restrict the latter to the decoupling limit. In this regime

$$
\mathcal{A}_{H}^{(5)} \rightarrow 4 \pi^{3} \frac{T_{L} T_{R}}{T_{H}} Q_{1} Q_{5},
$$

while all the velocities become lightlike (3.14), so $\varpi_{L, R}$ and $\varpi$ are identical quantities in both sides of the correspondence. Then we find

$$
\sigma_{\ell, p, m_{R, L}}^{\mathrm{CFT}}=\frac{\hat{\mathcal{V}}_{\ell}}{(\ell+1)^{2}} \sigma_{\ell, p, m_{R, L}}^{\text {sugra }}
$$

So the decay rates agree remarkably well, and it would only remain to check that a computation from first principles of $\hat{V}_{\ell}$, which is beyond the scope of this paper, yields a perfect match. Taking the limit $T_{R} \rightarrow 0$ we find the decay via superradiant emission of the ergo-cold black hole (3.57).

\section{CONCLUSIONS}

The recent progress in the microphysics of black holes is making it clear that the gravitational description of a microscopic system with a coarse-grain statistical degeneracy must exhibit a horizon - this may require higherderivative corrections if the degeneracy scales too slowly with the mass. In this paper our aim has been to find the microscopic origin of the ergoregion in rotating black holes. Cold ergoregions provide a particularly clean test bed, since they can only emit superradiant modes. What we have found is that, in order for a superradiant ergoregion to be present, the microscopic state must allow the annihilation of spin carriers to emit a bulk mode. If the system is at zero temperature, then these spin carriers must necessarily enter any interaction leading to bulk emission. The superradiant frequency bound follows then from the bound that the chemical potential sets on the energies of excitation charged under its canonically conjugated spin. All these features are transparent in the $1+1$ CFTs we have considered, in which the angular momentum is carried by fermions in (at least) one sector, while the other sector must also contain some excitations. This specific system has provided us with a simple, elementary derivation of the superradiant bound (1.1) without a detailed evaluation of absorption rates, which makes manifest the fundamental role played by the Fermi-Dirac statistics of the spincarrying degrees of freedom.

It seems likely that the basic features of our microscopic picture are also valid for any other gravitating object with a cold ergoregion. The most familiar of these is the extremal Kerr black hole. Reference [9] exhibited in a striking way how the absorption rates from a Kerr black hole contained hints of a CFT description. That this CFT must contain fermions as the carriers of angular momentum seems difficult to dispense with, if one wants to account for superradiant emission. Indeed, microscopic models for the extremal Kerr and five-dimensional Myers-Perry black holes have been proposed [4-6]. These black holes are mapped, through symmetries and dualities, to fourdimensional black holes of the kind we have discussed in Sec. II C. So the presence of superradiant emission in these neutral black holes is understood, at least qualitatively, in the same terms we have discussed: a filled Fermi sea in one sector of the dual CFT. The quantitative recovery of the superradiant bound is nevertheless not expected, since these neutral black holes suffer nontrivial renormalizations of their masses and energy levels (though not of their entropies) as a function of the coupling.

Systems with cold ergoregions which are not U-dual to these black holes are perhaps of more interest to test the applicability of our ideas about the microphysics of superradiance. An instance of this are the extremal rotating black rings with a dipole, in particular, those in which the dipole charge corresponds to a fundamental string and the extremal limit is singular. The microscopic description of this dipole ring has been described recently in [37], and argued to possess the right properties to fit our picture for a superradiating system: a zero-temperature sector with angular momentum carriers, which can interact with excitations from another sector and emit a spinning closed string into the bulk. Note, though, that in the system 
in [37] the angular momentum is not carried by a Fermi sea but by a bosonic coherent state.

All these ergo-cold black holes provide, in a sense, cleaner laboratories for the study of quantum emission from a black hole than do nonextremal black holes. Since one of their sectors is in a ground state, they are in a purer, less mixed state than nonextremal systems. But still, their other sector is in a mixed, thermal ensemble. Therefore it would be very interesting to consider states of the CFT such that both sectors are in pure states but nevertheless they can interact and decay by bulk emission. One such example is provided by the nonsupersymmetric smooth supergravity solitons with D1-D5-P charges in [29]. On the microscopic side, they correspond to nonchiral spectral flows of the Neveu-Schwarz ground state to non-BPS states in the Ramond sector. The states have both sectors containing only spin-carrying fermions. So we see that an interaction between the two sectors will result into the emission of a spinning bulk scalar. Following the overall picture proposed in this paper, superradiance is naturally expected. Indeed, these supergravity solitons have ergoregions (but not horizons) that have been shown to exhibit a superradiant instability [38]. A correspondence between the two pictures of the decay of precisely this type has been worked out in detail very recently in [39], and conforms to the overall ideas we have proposed.

\section{ACKNOWLEDGMENTS}

It is a pleasure to acknowledge stimulating discussions with Pau Figueras. This work has been supported in part by DURSI 2005 SGR 00082, CICYT FPA 2004-04582-C0202, FCT PTDC/FIS/ 64175/2006, and the European Community FP6 program MRTN-CT-2004-005104. O. J.C.D. acknowledges financial support provided by the European Community through the Intra-European Marie Curie contract MEIF-CT-2006-038924. A. M. was partially supported by a FPU grant from MEC (Spain).

\section{APPENDIX A: THE NEAR-HORIZON SIGNATURE OF SUPERRADIANCE}

It is natural to expect that the near-horizon geometry of the black hole, which encodes in a dual manner the CFT description, contains information about the possibility or not of superradiance. In the dual CFT, superradiance refers to an interaction between the CFT and a bulk scalar. The latter appears when the near-horizon geometry is not fully decoupled from asymptotic infinity and therefore disappears in the strict decoupling limit. Nevertheless, it would seem natural that the near-horizon geometry could still encode a signature that anticipates the existence of superradiant phenomena in the full geometry. An effect of this kind was identified in [18] for the extremal Kerr black hole, which is the simplest example of an ergo-cold black hole. From the study of scalar propagation in the near-horizon geometry, they could indeed identify an effect that signals superradiance in the Kerr solution. In this appendix we show how this same effect is present in our ergo-cold black hole, but not in the BMPV solution.

\section{Near-horizon geometry}

Take the black hole solutions of the D1-D5-P system described in (3.1). To obtain their near-horizon geometry we introduce

$$
r^{2}=r_{+}^{2}+\varepsilon \rho, \quad \tau=\gamma \frac{t}{\varepsilon},
$$

where $\gamma$ is a constant to be defined later, and we take the $\varepsilon \rightarrow 0$ limit. To avoid divergencies of the type $1 / \varepsilon$ and $1 / \varepsilon^{2}$, this coordinate transformation must be accompanied by a shift in the circle and angular directions,

$$
\begin{gathered}
y=\tilde{y}+V_{H} \frac{t}{\varepsilon}, \quad \phi=\tilde{\phi}+\Omega_{\phi} \frac{t}{\varepsilon}, \\
\psi=\tilde{\psi}+\Omega_{\psi} \frac{t}{\varepsilon},
\end{gathered}
$$

where $\Omega_{\phi}, \Omega_{\psi}, V_{H}$ represent the horizon angular velocities defined in (3.8) and (3.13). With the shift (A2), the Killing vector $\partial / \partial_{t}$ becomes tangent to the horizon, i.e., the new coordinates corotate with the horizon. Next, we just write the near-horizon limit of the extreme black hole metrics (in the end of this appendix we comment on the nonextreme cases), since the near-horizon dilaton and RR fields are not important for our discussion.

(i) Near-horizon geometry of the BPS black hole.

In this case one has $\Omega_{\phi, \psi}=0$ and $\gamma=\ell_{3}^{2} \sqrt{Q_{p}} / 2$ and one gets (dropping the $\sim$ in the angular coordinates),

$$
\begin{aligned}
d s_{\mathrm{NH}}^{2}= & \frac{\ell_{3}^{2}}{4}\left(-\rho^{2} d \tau^{2}+\frac{d \rho^{2}}{\rho^{2}}\right)+\ell_{3}^{2}\left(d \theta^{2}+\sin ^{2} \theta d \phi^{2}\right. \\
& \left.+\cos ^{2} \theta d \psi^{2}\right)+\frac{Q_{p}}{\ell_{3}^{2}}\left(d y+\frac{\ell_{3}^{2} \rho}{2 \sqrt{Q_{p}}} d \tau\right)^{2} \\
& +\frac{2 J_{\phi}}{\ell_{3}^{2}} d y\left(\sin ^{2} \theta d \phi+\cos ^{2} \theta d \psi\right),
\end{aligned}
$$

where $\ell_{3}^{2}=\sqrt{Q_{1} Q_{5}}$.

(ii) Near-horizon geometry of the ergo-cold black hole. One has $\Omega_{\phi}=\Omega_{\psi}$. We restrict our attention to the simplest case with $a_{1}=a_{2}$. This case contains all the features that are crucial for our study and does not loose any important information, while avoiding noninsightful factors.

One gets, with $\gamma=-\left[2 a^{3}\left(c_{1} c_{5} c_{p}+s_{1} s_{5} s_{p}\right)\right]^{-1}$ (and dropping the $\sim$ in the angular coordinates), 


$$
\begin{aligned}
d s_{\mathrm{NH}}^{2}= & \frac{K_{0}}{4}\left(-\rho^{2} d \tau^{2}+\frac{d \rho^{2}}{\rho^{2}}\right)+K_{0} d \theta^{2}+K\left(\sin ^{2} \theta d \phi+\cos ^{2} \theta d \psi+P \rho d \tau\right)^{2}+K_{0} \sin ^{2} \theta(d \phi+P \rho d \tau)^{2} \\
& +K_{0} \cos ^{2} \theta(d \psi+P \rho d \tau)^{2}+K_{y}\left[d y+K_{t y} \rho d \tau+P_{\phi y}\left(\sin ^{2} \theta d \phi+\cos ^{2} \theta d \psi\right)\right]^{2},
\end{aligned}
$$

where $K_{0}, K, P, K_{y}, K_{t y}, K_{\phi y}$ are constants given in terms of the black hole parameters $a, \delta_{1,5, p}$ by

$$
\begin{gathered}
K_{0}=2 a^{2} \sqrt{\cosh \left(2 \delta_{1}\right) \cosh \left(2 \delta_{5}\right)}, \quad K=\frac{2 a^{2}\left[1-2 \operatorname{sech}\left(2 \delta_{p}\right)\left(s_{1} s_{5} c_{p}-c_{1} c_{5} s_{p}\right)^{2}\right]}{\sqrt{\cosh \left(2 \delta_{1}\right) \cosh \left(2 \delta_{5}\right)}}, \\
P=-\frac{1+\cosh \left(2 \delta_{1}\right) \cosh \left(2 \delta_{5}\right)+\cosh \left(2 \delta_{1}\right) \cosh \left(2 \delta_{p}\right)+\cosh \left(2 \delta_{5}\right) \cosh \left(2 \delta_{p}\right)}{8\left(s_{1} s_{5} s_{p}+c_{1} c_{5} c_{p}\right)^{2}}, \quad K_{y}=\frac{\cosh \left(2 \delta_{p}\right)}{\sqrt{\cosh \left(2 \delta_{1}\right) \cosh \left(2 \delta_{5}\right)}}, \\
P_{\phi y}=-2 a\left(s_{1} s_{5} c_{p}-c_{1} c_{5} s_{p}\right) \operatorname{sech}\left(2 \delta_{p}\right), \quad P_{t y}=-a\left(s_{1} s_{5} c_{p}+c_{1} c_{5} s_{p}\right) \operatorname{sech}\left(2 \delta_{p}\right) .
\end{gathered}
$$

When $a_{1} \neq a_{2}$, there are overall $\theta$-dependent multiplicative factors both on the $\mathrm{AdS}_{2}$ and fibred $S^{3}$ parts of the metric. They play no fundamental role in the analysis that we do next.

The key observation in (A4) is that the cross terms between the time coordinate $\tau$ and the angular coordinates $\phi, \psi$, are linear in the radial coordinate $\rho$ in the case of the black hole with ergoregion. On the other hand, when the ergoregion is absent, the radial dependence in the cross terms is also not present. This feature plays an important role in the near-horizon superradiant analysis that we do next.

\section{The Bardeen-Horowitz signature of superradiance}

In this section we identify and justify the superradiant signature in a near-horizon geometry. We refer to this as the Bardeen-Horowitz signature, since the feature that we will describe was first identified by these authors in the extremal Kerr solution [18]. We will initially focus our analysis on the near-horizon geometry (A4) of the ergocold black hole. We will single out the factor responsible for superradiance in this case. Then we will observe that this factor is absent when the ergoregion is not present, and, in particular, in the BPS case.

Take (A4). The following analysis gets simplified if we carry dimensional reduction along $y$ (again we will take waves with no momentum along the $T^{4}$, so this plays no role in the discussion). This yields ${ }^{6}$

\footnotetext{
${ }^{6}$ We absorb a factor of $K_{y}^{-1}$ in the left-hand side that comes from the KK dilaton (which being constant plays no role): $d s_{\mathrm{NH}(5)}^{2} \equiv K_{y}^{-1} d s_{\mathrm{NH}(5)}^{2}$. There is also a gauge field which is irrelevant for our purposes, and whose components are $A_{\tau}=$ $K_{t y} \rho, A_{\phi}=K_{\phi y} \sin ^{2} \theta, A_{\psi}=K_{\phi y} \cos ^{2} \theta$.
}

$$
\begin{aligned}
d s_{\mathrm{NH}(5)}^{2}= & \frac{K_{0}}{4}\left(-\rho^{2} d \tau^{2}+\frac{d \rho^{2}}{\rho^{2}}\right)+K_{0} d \theta^{2} \\
& +K\left(\sin ^{2} \theta d \phi+\cos ^{2} \theta d \psi+P \rho d \tau\right)^{2} \\
& +K_{0} \sin ^{2} \theta(d \phi+P \rho d \tau)^{2} \\
& +K_{0} \cos ^{2} \theta(d \psi+P \rho d \tau)^{2} .
\end{aligned}
$$

This five-dimensional metric is of the form $\mathrm{AdS}_{2} \times S^{3}$. We can introduce global $\mathrm{AdS}_{2}$ coordinates to cover the entire spacetime [18],

$$
\rho=\sqrt{1+x^{2}} \cos T+x, \quad \tau=\frac{\sqrt{1+x^{2}} \sin T}{\rho},
$$

whose ranges are $-\infty<T<\infty,-\infty<x<\infty$. To avoid new crossed terms between $S^{3}$ and $\mathrm{AdS}_{2}$ coordinates, we have to shift $\phi$ and $\psi[18]$,

$$
\phi, \psi=\tilde{\phi}, \tilde{\psi}+P \log \left[\frac{\cos T+x \sin T}{1+\sqrt{1+x^{2}} \sin T}\right] .
$$

In these global coordinates the metric (A6) reads

$$
\begin{aligned}
d s_{\mathrm{NH}(5)}^{2}= & \frac{K_{0}}{4}\left(-\left(1+x^{2}\right) d T^{2}+\frac{d x^{2}}{1+x^{2}}\right)+K_{0} d \theta^{2} \\
& +K\left(\sin ^{2} \theta d \tilde{\phi}+\cos ^{2} \theta d \tilde{\psi}+P x d T\right)^{2} \\
& +K_{0} \sin ^{2} \theta(d \tilde{\phi}+P x d T)^{2} \\
& +K_{0} \cos ^{2} \theta(d \tilde{\psi}+P x d T)^{2} .
\end{aligned}
$$

We now study the Klein-Gordon equation in this nearhorizon background (A9). Introducing the ansatz

$$
\Phi=e^{-i(w T-m \tilde{\phi}-n \tilde{\psi})} \Theta(\theta) X(x),
$$

the wave equation separates and yields 


$$
\begin{aligned}
\frac{1}{\sin 2 \theta} \frac{d}{d \theta}\left[\sin 2 \theta \frac{d \Theta}{d \theta}\right]+ & {\left[\Lambda-\frac{m^{2}}{\sin ^{2} \theta}-\frac{n^{2}}{\cos ^{2} \theta}\right] \Theta=0, } \\
\frac{d}{d x}\left[\left(1+x^{2}\right) \frac{d X}{d x}\right]+ & \frac{1}{4}\left[\frac{4[w+(m+n) P x]^{2}}{1+x^{2}}\right. \\
& \left.+\frac{K}{K+K_{0}}(m+n)^{2}-\Lambda\right] X=0,
\end{aligned}
$$

where $K_{0}, K$, and $P$ are defined in (A5). ${ }^{7}$

The radial equation presents an important feature. Indeed, apart from the contribution coming from the piece $(m+n) P x$, this radial equation is very similar to the equation describing perturbations in a pure $\mathrm{AdS}_{2}$ background [18]. That is, in (A11) we have $[w+(m+$ $n) P x]^{2}$ instead of $w^{2}$ that is present in the pure $\mathrm{AdS}_{2}$ case. The origin of this factor can be easily traced back and found to be due to the presence of the terms $P \rho d \tau$ in (A4); see the discussion at the end of Sec. A 1. We next discuss the implications of this property for the nearhorizon signature of superradiance.

In a WKB approximation the effective wave number for traveling waves obeying (A11), $k=-\frac{i}{X} \frac{d X}{d x}$, is

$$
\begin{aligned}
k= & \pm \frac{1}{4 \sqrt{1+x^{2}}}\left[\frac{4[w+(m+n) P x]^{2}}{1+x^{2}}\right. \\
& \left.+\frac{K}{K+K_{0}}(m+n)^{2}-\Lambda\right]^{1 / 2},
\end{aligned}
$$

from which follows the associated group velocity,

$$
\begin{aligned}
\frac{d w}{d k}= & \pm \frac{4\left(1+x^{2}\right)^{3 / 2}}{w+(m+n) P x}\left[\frac{[w+(m+n) P x]^{2}}{1+x^{2}}\right. \\
& \left.+\frac{K}{K+K_{0}}(m+n)^{2}-\Lambda\right]^{1 / 2} .
\end{aligned}
$$

\footnotetext{
${ }^{7}$ The separation constant is exactly $\Lambda=\ell(\ell+2)$ (this is a consequence of working with the $a_{1}=a_{2}$ case), and poses a bound on the other angular quantum numbers: $\ell \geq|m|+|n|$.
}

On the other hand, the phase velocity of the waves is $w / k$. As first observed in [18], in the context of the Kerr geometry, here the group and phase velocities can have opposite signs. For positive $(m+n) P$ this occurs when $x<\frac{w}{(m+n) P}$, while for negative $(m+n) P$ this is true when $x>\frac{w}{(m+n) P}$. An original argument from Press and Teukolsky [16] concludes that this defines the near-horizon superradiant regime. Indeed, the opposite sign between group and phase velocities of a wave in the vicinity of a horizon is responsible for the fundamental origin of superradiance. Classically, only ingoing waves are allowed to cross the horizon. The quantity that defines the physical direction of a wave is its group velocity rather than its phase velocity. So the classical absorption of incident waves is described by imposing a negative group velocity as a boundary condition. Note however that in the near-horizon superradiant regime above mentioned, the associated phase velocity is positive and so waves appear as outgoing to an inertial observer at spatial infinity. Thus, energy is in fact being extracted, i.e., superradiance is active [16].

At this point, we make a contact with the other extreme case and with the discussion in the end of Sec. A 1. For the BPS black hole, there is no radial dependence in the cross terms between the time and angular coordinates in its nearhorizon geometry (A3). As a consequence, there is no linear term in the frequency in the wave equation associated with this background. But this implies that group and phase velocities always have the same sign in this background. Thus there is no available room for a superradiant regime in the near-horizon geometries of extreme black holes without ergoregion. Finally note that in a general nonextreme black hole the situation is quite similar to the ergo-cold black hole in what concerns the issue discussed in this appendix.
[1] A. Strominger and C. Vafa, Phys. Lett. B 379, 99 (1996).

[2] G. T. Horowitz and A. Strominger, Phys. Rev. Lett. 77, 2368 (1996).

[3] J. C. Breckenridge, D. A. Lowe, R. C. Myers, A. W. Peet, A. Strominger, and C. Vafa, Phys. Lett. B 381, 423 (1996).

[4] R. Emparan and G. T. Horowitz, Phys. Rev. Lett. 97, 141601 (2006).

[5] R. Emparan and A. Maccarrone, Phys. Rev. D 75, 084006 (2007).

[6] G. T. Horowitz and M. M. Roberts, Phys. Rev. Lett. 99, 221601 (2007).
[7] S. R. Das and S. D. Mathur, Nucl. Phys. B478, 561 (1996).

[8] S. R. Das and S.D. Mathur, Nucl. Phys. B482, 153 (1996).

[9] J. M. Maldacena and A. Strominger, Phys. Rev. D 56, 4975 (1997).

[10] S. D. Mathur, Nucl. Phys. B514, 204 (1998).

[11] S. S. Gubser, Phys. Rev. D 56, 4984 (1997).

[12] Ya. B. Zel'dovich, Pis'ma Zh. Eksp. Teor. Fiz. 14, 270 (1971) [JETP Lett. 14, 180 (1971)]; Zh. Eksp. Teor. Fiz. 62, 2076 (1972) [Sov. Phys. JETP 35, 1085 (1972)].

[13] C. W. Misner, Phys. Rev. Lett. 28, 994 (1972). 
[14] A. A. Starobinsky, Sov. Phys. JETP 37, 28 (1973); A. A. Starobinsky and S. M. Churilov, Sov. Phys. JETP 38, 1 (1973).

[15] J. D. Bekenstein, Phys. Rev. D 7, 949 (1973).

[16] W. H. Press and S.A. Teukolsky, Astrophys. J. 185, 649 (1973).

[17] W. G. Unruh, Phys. Rev. D 10, 3194 (1974); 14, 3251 (1976).

[18] J. M. Bardeen and G. T. Horowitz, Phys. Rev. D 60, 104030 (1999).

[19] V. Cardoso, O. J. C. Dias, J. P. S. Lemos, and S. Yoshida, Phys. Rev. D 70, 044039 (2004); 70, 049903(E) (2004).

[20] M. Cvetic and F. Larsen, Phys. Rev. D 56, 4994 (1997).

[21] M. Cvetic and F. Larsen, Nucl. Phys. B531, 239 (1998).

[22] S. S. Gubser, arXiv:hep-th/9908004.

[23] J. R. David, G. Mandal, and S. R. Wadia, Phys. Rep. 369, 549 (2002).

[24] C. G. Callan and J. M. Maldacena, Nucl. Phys. B472, 591 (1996).

[25] J. C. Breckenridge, R. C. Myers, A. W. Peet, and C. Vafa, Phys. Lett. B 391, 93 (1997).

[26] G. W. Gibbons, Commun. Math. Phys. 44, 245 (1975).

[27] M. Cvetic and D. Youm, Nucl. Phys. B476, 118 (1996); D. Youm, Phys. Rep. 316, 1 (1999).
[28] S. Giusto, S. D. Mathur, and A. Saxena, Nucl. Phys. B701, 357 (2004).

[29] V. Jejjala, O. Madden, S. F. Ross, and G. Titchener, Phys. Rev. D 71, 124030 (2005).

[30] E. Berti, V. Cardoso, and M. Casals, Phys. Rev. D 73, 024013 (2006); 73, 109902(E) (2006).

[31] V. Cardoso, O. J. C. Dias, and R. C. Myers, Phys. Rev. D 76, 105015 (2007).

[32] M. Abramowitz and A. Stegun, Handbook of Mathematical Functions (Dover Publications, New York, 1970).

[33] C. G. Callan, S. S. Gubser, I. R. Klebanov, and A. A. Tseytlin, Nucl. Phys. B489, 65 (1997).

[34] V. Cardoso and J.P.S. Lemos, Phys. Lett. B 621, 219 (2005).

[35] O. J. C. Dias, Phys. Rev. D 73, 124035 (2006).

[36] V. Balasubramanian, J. de Boer, E. Keski-Vakkuri, and S.F. Ross, Phys. Rev. D 64, 064011 (2001). J. M. Maldacena and L. Maoz, J. High Energy Phys. 12 (2002) 055.

[37] J. J. Blanco-Pillado, R. Emparan, and A. Iglesias, J. High Energy Phys. 01 (2008) 014.

[38] V. Cardoso, O. J. C. Dias, J. L. Hovdebo, and R. C. Myers, Phys. Rev. D 73, 064031 (2006).

[39] B. D. Chowdhury and S. D. Mathur, arXiv:0711.4817. 